\title{
Noncommutative Configuration Space. Classical and Quantum Mechanical Aspects
}

\author{
F. J. Vanhecke, C. Sigaud, and A. R. da Silva \\ Instituto de Física, Instituto de Matemática, UFRJ, Rio de Janeiro, Brazil
}

Received on 17 October, 2005

\begin{abstract}
In this work we examine noncommutativity of position coordinates in classical symplectic mechanics and its quantisation. In coordinates $\left\{q^{i}, p_{k}\right\}$ the canonical symplectic two-form is $\omega_{0}=d q^{i} \wedge d p_{i}$. It is well known in symplectic mechanics $[5,6,9]$ that the interaction of a charged particle with a magnetic field can be described in a Hamiltonian formalism without a choice of a potential. This is done by means of a modified symplectic twoform $\omega=\omega_{0}-e \mathbf{F}$, where $e$ is the charge and the (time-independent) magnetic field $\mathbf{F}$ is closed: $\mathbf{d F}=0$. With this symplectic structure, the canonical momentum variables acquire non-vanishing Poisson brackets: $\left\{p_{k}, p_{l}\right\}=$ $e F_{k l}(q)$. Similarly a closed two-form in $p$-space $\mathbf{G}$ may be introduced. Such a dual magnetic field $\mathbf{G}$ interacts with the particle's dual charge $r$. A new modified symplectic two-form $\omega=\omega_{0}-e \mathbf{F}+r \mathbf{G}$ is then defined. Now, both $p$ - and $q$-variables will cease to Poisson commute and upon quantisation they become noncommuting operators. In the particular case of a linear phase space $\mathbf{R}^{2 N}$, it makes sense to consider constant $\mathbf{F}$ and $\mathbf{G}$ fields. It is then possible to define, by a linear transformation, global Darboux coordinates: $\left\{\xi^{i}, \pi_{k}\right\}=\delta^{i}{ }_{k}$. These can then be quantised in the usual way $\left[\widehat{\xi}^{i}, \widehat{\pi}_{k}\right]=i \hbar \delta^{i}$. The case of a quadratic potential is examined with some detail when $N$ equals 2 and 3 .
\end{abstract}

Keywords: Noncommutativity; Symplectic mechanics; Quantization

\section{INTRODUCTION}

The idea to consider non vanishing commutation relations between position operators $[\mathbf{x}, \mathbf{y}]=i \ell^{2}$, analogous to the canonical commutation relations between position and conjugate momentum $\left[\mathbf{x}, \mathbf{p}_{x}\right]=i \hbar$, is ascribed to Heisenberg, who saw there a possibility to introduce a fundamental lenght $\ell$ which might control the short distance singularities of quantum field theory. However, noncommutativity of coordinates appeared first nonrelativistically in the work of Peierls [2] on the diamagnetism of conduction electrons. In the limit of a strong magnetic field in the $z$-direction, the gap between Landau levels becomes large and, to leading order, one obtains $[\mathbf{x}, \mathbf{y}]=i \hbar c / e B$. In relativistic quantum mechanics, noncommutativity was first examined in 1947 by Snyder [3] and, in the last five years, inspired by string and brane-theory, many papers on field theory in noncommutative spaces appeared in the physics literature. The apparent unitarity problem related to time-space noncommutativity in field theory was studied and solved in [10]. Also (nonrelativistic) quantum mechanics on noncommutative twodimensional spaces has been examined more thorougly in the recent years: [11-16]. The above mentionned unitarity problem in quantum physics is also examined in Balachandran et al. [17].

In this work we discuss noncommutativity of configuration space $Q$ in classical mechanics on the cotangent bundle $T^{*}(Q)$ and its canonical quantisation in the most simple case. In section II we review the classical theory of a non relativistic particle interacting with a time-independent magnetic field $\mathbf{F}=1 / 2 F_{i j}(q) d q^{i} \wedge d q^{j} ; \mathbf{d F}=0$. This is done in every textbook introducing a potential in a Lagrangian formalism. The Legendre transformation defines then the Hamiltonian and the canonical symplectic two-form $d q^{i} \wedge d p_{i}$ implements the corresponding Hamiltonian vector field. We also recall the less well known procedure of avoiding the introduction of a potential using a modified symplectic structure: $\omega=d q^{i} \wedge d p_{i}-e \mathbf{F}$. The coupling with the charge $e$ is hidden in the symplectic structure and does not show up in the Hamiltonian: $H_{0}(q, p)=$ $\delta^{k l} p_{k} p_{l} / 2 m+\mathcal{V}(q)$. In section III, a closed two-form in $p$ space, the dual field: $\mathbf{G}=1 / 2 G^{k l}(p) d p_{k} \wedge d p_{l}$, is added to the symplectic structure $\omega=d q^{i} \wedge d p_{i}-e \mathbf{F}+r \mathbf{G}$, where $r$ is a dual charge.

Such an approach with a modified symplectic structure has been previously considered by Duval and Horvathy [11, 14] emphasizing the $N=2$-dimensional case in connection with the quantum Hall effect. We should also mention Plyushchay's interpretation [18] of such a dual charge $r$ when $N=2$ as the anyon spin. Considering here an arbitrary number of dimensions $N$, no such interpretation of $r$ is assumed. The crucial point is that, now, both $p$ - and $q$-variables cease to Poisson commute and upon quantisation they should become noncommuting operators. In the particular case of a linear phase space $\mathbf{R}^{2 N}$, it makes sense to consider constant $\mathbf{F}$ and $\mathbf{G}$ fields. It is then possible to define global Darboux coordinates with Poisson brackets $\left\{\xi^{i}, \pi_{k}\right\}=\delta^{i}{ }_{k}$. These can then be quantised uniquely [1] in the usual way: $\left[\widehat{\xi}^{i}, \widehat{\pi}_{k}\right]=i \hbar \delta^{i}{ }_{k}$. However, in general, the dynamics become non-linear and there is no guarantee that the Hamiltonian vector field is complete. It is then not trivial to quantise the Hamiltonian, which becomes nonlocal. However, for a linear or quadratic Hamiltonian, this is possible and it is seen that the noncommutativity generates a magnetic moment type interaction. The cases $N=2$ and $N=3$ are discussed in detail in section IV. In section V we examine the problem of symmetries in the modified symplectic manifold. Finally, in section VI general comments are made and further developments are suggested. In appendix A we recall 
basic notions in symplectic geometry and in appendix B we give a brief account of the Gotay-Nester-Hinds algorithm [7] for constrained Hamiltonian systems.

\section{NON RELATIVISTIC PARTICLE INTERACTING WITH A TIME-INDEPENDENT MAGNETIC FIELD}

A particle of mass $m$ and charge $e$, with potential energy $\mathcal{V}$, moving in a Euclidean configuration space $Q$, with cartesian coordinates $q^{i}$, interacts with a (time-independent) magnetic field given by a closed two-form $\mathbf{F}(q)=\frac{1}{2} F_{i j}(q) \mathbf{d} q^{i} \wedge \mathbf{d} q^{j}$. The dynamics is given by the Laplace equation:

$$
m \frac{\mathbf{d}^{2} q^{i}}{\mathbf{d} t^{2}}=\delta^{i j}\left(e F_{j k}(q) \frac{\mathbf{d} q^{k}}{\mathbf{d} t}-\frac{\partial \mathcal{V}(q)}{\partial q^{j}}\right) .
$$

Assuming $Q$ to be Euclidean avoids topological subtleties, so that there exists a global potential one-form $\mathbf{A}(q)=A_{i}(q) \mathbf{d} q^{i}$ such that $\mathbf{F}=\mathbf{d A}$. A global Lagrangian formalism can then be established with a Lagrangian function on the tangent bundle $\{\tau: T(Q) \rightarrow Q\}:$

$$
\mathcal{L}(q, \dot{q})=\frac{1}{2} m \delta_{i j} \dot{q}^{i} \dot{q}^{j}+e \dot{q}^{i} A_{i}(q)-\mathcal{V}(q)
$$

The Euler-Lagrange equation is obtained as:

$$
\begin{aligned}
0 & =\frac{\partial \mathcal{L}}{\partial q^{i}}-\frac{\mathbf{d}}{\mathbf{d} t} \frac{\partial \mathcal{L}}{\partial \dot{q}^{i}}=-\frac{\partial \mathcal{V}}{\partial q^{i}}+e \dot{q}^{k} \frac{\partial A_{k}(q)}{\partial q^{i}}-\frac{\mathbf{d}}{\mathbf{d} t}\left(m \delta_{i j} \dot{q}^{j}+e A_{i}(q)\right) \\
& =-\frac{\partial \mathcal{V}}{\partial q^{i}}+e \dot{q}^{k}\left(\frac{\partial A_{k}(q)}{\partial q^{i}}-\frac{\partial A_{i}(q)}{\partial q^{k}}\right)-m \frac{\mathbf{d}}{\mathbf{d} t} \delta_{i j} \dot{q}^{j} \\
& =-\frac{\partial \mathcal{V}}{\partial q^{i}}+e \mathbf{F}_{i k}(q) \dot{q}^{k}-m \delta_{i j} \ddot{q}^{j}
\end{aligned}
$$

and coincides with the Laplace equation (II.1).

The Legendre transform

$$
\left(q^{i}, \dot{q}^{j}\right) \rightarrow\left(q^{i}, p_{k}=\frac{\partial \mathcal{L}}{\partial \dot{q}^{k}}=m \delta_{k l} \dot{q}^{l}+e A_{k}(q)\right),
$$

defines the Hamiltonian on the cotangent bundle $\left\{T^{*}(Q) \stackrel{\kappa}{\longrightarrow}\right.$ Q\}:

$$
\begin{gathered}
\mathcal{H}_{\mathbf{A}}(q, p)=-\mathcal{L}(q, \dot{q})+p_{i} \dot{q}^{i}= \\
\frac{1}{2 m} \delta^{k l}\left(p_{k}-e A_{k}(q)\right)\left(p_{l}-e A_{l}(q)\right)+\mathcal{V}(q) .
\end{gathered}
$$

With the canonical symplectic two-form

$$
\omega_{0}=\mathbf{d} q^{i} \wedge \mathbf{d} p_{i},
$$

the Hamiltonian vector field of $\mathcal{H}_{\mathbf{A}}$ is:

$\mathbf{X}_{\mathcal{H}}=\frac{\delta^{i j}}{m}\left(p_{j}-e A_{j}\right) \frac{\partial}{\partial \mathbf{q}^{i}}+\left(\frac{e}{m} \delta^{k l} \frac{\partial A_{k}}{\partial q^{i}}\left(p_{l}-e A_{l}\right)-\frac{\partial \mathcal{V}}{\partial q^{i}}\right) \frac{\partial}{\partial \mathbf{p}_{i}}$

Its integral curves are solutions of:

$$
\frac{\mathbf{d} q^{i}}{\mathbf{d} t}=\frac{\delta^{i j}}{m}\left(p_{j}-e A_{j}\right), \frac{\mathbf{d} p_{i}}{\mathbf{d} t}=\frac{e}{m} \delta^{k l} \frac{\partial A_{k}}{\partial q^{i}}\left(p_{l}-e A_{l}\right)-\frac{\partial \mathcal{V}}{\partial q^{i}},
$$

which is again equivalent to (II.1).
If the second de Rham cohomology were not trivial, $H_{d R}^{2}(Q) \neq 0$, there is no global potential $\mathbf{A}$ and a local Lagrangian formalism is needed. This can be done enlarging the configuration space $Q$ to the total space $P$ of a principal $U(1)$ bundle over $Q$ with a connection, given locally by $\mathbf{A}[19]$. This can be avoided using a global Hamiltonian formalism[20] in the cotangent bundle $T^{*}(Q)$ using a modified symplectic twoform:

$$
\omega=\omega_{0}-e \mathbf{F}=\mathbf{d} q^{i} \wedge \mathbf{d} p_{i}-\frac{1}{2} e F_{i j}(q) \mathbf{d} q^{i} \wedge \mathbf{d} q^{j}
$$

and a "charge-free" Hamiltonian:

$$
\mathcal{H}_{0}(p, q)=\frac{1}{2 m} \delta^{k l} p_{k} p_{l}+\mathcal{V}(q)
$$

The Hamiltonian vector fields corresponding to an observable $f(q, p)$ are now defined relative to $\omega$ as ${ }^{l} \mathbf{X}_{f}^{\mathbf{F}} \boldsymbol{\omega}=\mathbf{d} f$ and given by:

$$
\mathbf{X}_{f}^{\mathbf{F}}=\frac{\partial f}{\partial p_{i}} \frac{\partial}{\partial \mathbf{q}^{i}}-\left(\frac{\partial f}{\partial q^{l}}+\frac{\partial f}{\partial p_{k}} e F_{k l}(q)\right) \frac{\partial}{\partial \mathbf{p}_{l}}
$$

With the Hamiltonian $\mathcal{H}_{0}$, the dynamics are again given by the Laplace equation (II.1) in the form:

$$
\frac{\mathbf{d} q^{i}}{\mathbf{d} t}=\frac{\delta^{i j}}{m} p_{j} ; \frac{\mathbf{d} p_{l}}{\mathbf{d} t}=-\delta^{k i}\left(\frac{\partial \mathcal{V}}{\partial q^{i}}+\frac{e}{m} p_{i} F_{k l}(q)\right) .
$$


The Poisson brackets, relative to the symplectic structure II.5, are:

$$
\{f, g\}=\frac{\partial f}{\partial q^{i}} \frac{\partial g}{\partial p_{i}}-\frac{\partial f}{\partial p_{i}} \frac{\partial g}{\partial q^{i}}+\frac{\partial f}{\partial p_{k}} e F_{k l}(q) \frac{\partial g}{\partial p_{l}} .
$$

In particular, the coordinates themselves have Poisson brackets:

$$
\begin{aligned}
& \left\{q^{i}, q^{j}\right\}=0,\left\{q^{i}, p_{l}\right\}=\delta_{l}^{i}, \\
& \left\{p_{k}, q^{j}\right\}=-\delta_{k}^{j},\left\{p_{k}, p_{l}\right\}=e F_{k l}(q) .
\end{aligned}
$$

Obviously, the meaning of the $\{q, p\}$ variables in (II.3) and (II.5) are different. However both formalisms $\left(\omega_{0}, \mathcal{H}_{\mathbf{A}}\right)$ and $\left(\omega, \mathcal{H}_{0}\right)$ lead to the same equations of motion and thus, they must be equivalent. Indeed, in each open set $U$ homeomorphic to $\mathbf{R}^{6}$, the vanishing $\mathbf{d F}=0$ implies the existence of $\mathbf{A}$ such that $\mathbf{F}=\mathbf{d A}$ in $U$ and, locally:

$$
\omega=\mathbf{d} q^{i} \wedge \mathbf{d} p_{i}-\frac{1}{2} e F_{i j} \mathbf{d} q^{i} \wedge \mathbf{d} q^{j}=-\mathbf{d}\left[\left(p_{i}+e A_{i}\right) \mathbf{d} q^{i}\right] .
$$

Thus there exist local Darboux coordinates:

$$
\xi^{i}=q^{i}, \pi_{k}=p_{k}+e A_{k}(q)
$$

such that $\omega=\mathbf{d} \xi^{i} \wedge \mathbf{d} \pi_{i}$, which is the form (II.3).

The dynamics defined by the Hamiltonian $\mathcal{H}_{0}(q, p)=$ $p^{2} / 2 m+\mathcal{V}(q)$, with symplectic two-form $\omega$, is equivalent to the dynamics defined by the Hamiltonian $\mathcal{H}_{\mathbf{A}}(\xi, \pi)=(\pi-$ $e A(\xi))^{2} / 2 m+\mathcal{V}(\xi)$ and canonical symplectic structure $\omega=$ $\mathbf{d} \xi^{i} \wedge \mathbf{d} \pi_{i}$. Equivalence is trivial since both symplectic twoforms are equal, but expressed in different coordinates $\{q, p\}$ and $\{\xi, \pi\}$, related by (II.9). It seems worthwhile to note that a gauge transformation $\mathbf{A} \rightarrow \mathbf{A}^{\prime}=\mathbf{A}+\operatorname{grad} \phi$ corresponds to a change of Darboux coordinates

$$
\left\{\xi^{i}, \pi_{k}\right\} \Rightarrow\left\{\xi^{i \prime}=\xi^{i}, \pi_{k}^{\prime}=\pi_{k}+e \partial_{k} \phi\right\},
$$

i.e. a symplectic transformation.

\section{NONCOMMUTATIVE COORDINATES}

Let us consider an affine configuration space $Q=\mathbf{A}^{N}$ so that points of phase space, identified with $\mathcal{M} \doteq \mathbf{R}^{2 N}=$ $\mathbf{R}_{q}^{N} \times \mathbf{R}_{p}^{N}$, may be given by linear coordinates $(q, p)$. Together with the (usual) magnetic field $\mathbf{F}$, we may introduce a (dual) magnetic field $\mathbf{G}=1 / 2 G^{k l}(p) \mathbf{d} p_{k} \wedge \mathbf{d} p_{l}$, a closed two-form, $\mathbf{d G}=0$, in $\mathbf{R}_{p}^{n}$ space. Let $e$ be the usual electric charge and $r$, a dual charge, which couples the particle with $\mathbf{F}$ and $\mathbf{G}$. Consider the closed two-form:

$$
\begin{aligned}
\omega & =\omega_{0}-e \mathbf{F}+r \mathbf{G} \\
& =\mathbf{d} q^{i} \wedge \mathbf{d} p_{i}-\frac{1}{2} e F_{i j}(q) \mathbf{d} q^{i} \wedge \mathbf{d} q^{j}+\frac{1}{2} r G^{k l}(p) \mathbf{d} p_{k} \wedge \mathbf{d} p_{l} .
\end{aligned}
$$

In matrix notation this two-form (III.1) is represented as:

$$
\begin{aligned}
(\Omega) & =\left(\begin{array}{cc}
-e \mathbf{F} & \mathbf{1} \\
-\mathbf{1} & +r \mathbf{G}
\end{array}\right) \\
& =\left(\begin{array}{cc}
0 & \mathbf{1} \\
\mathbf{1} & +r \mathbf{G}
\end{array}\right)\left(\begin{array}{cc}
-\Psi & 0 \\
0 & \mathbf{1}
\end{array}\right)\left(\begin{array}{cc}
\mathbf{1} & 0 \\
-e \mathbf{F} & \mathbf{1}
\end{array}\right) \\
& =\left(\begin{array}{cc}
e \mathbf{F} & \mathbf{1} \\
\mathbf{1} & 0
\end{array}\right)\left(\begin{array}{cc}
-\mathbf{1} & 0 \\
0 & \Phi
\end{array}\right)\left(\begin{array}{cc}
\mathbf{1} & -r \mathbf{G} \\
0 & \mathbf{1}
\end{array}\right) .
\end{aligned}
$$

where[21] $\Phi=(\mathbf{1}-e \mathbf{F} r \mathbf{G}) ; \Psi=(\mathbf{1}-r \mathbf{G} e \mathbf{F})$.

The fundamental Hamiltonian equation $\iota_{\mathbf{X}} \boldsymbol{\omega}=\mathbf{d} f$, in (A.1), reads:

$$
\left(X^{i}-r G^{i j} X_{j}\right) \mathbf{d} p_{i}-\left(X_{k}-e F_{k l} X^{l}\right) \mathbf{d} q^{k}=\frac{\partial f}{\partial q^{k}} \mathbf{d} q^{k}+\frac{\partial f}{\partial p_{i}} \mathbf{d} p_{i} .
$$

This can be rewritten as

$$
\left(\frac{\partial f}{\partial p_{i}}-r G^{i j} \frac{\partial f}{\partial q^{j}}\right)=\Psi_{j}^{i} X^{j} ;\left(\frac{\partial f}{\partial q^{k}}-e F_{k l} \frac{\partial f}{\partial p^{l}}\right)=-\Phi_{k}^{l} X_{l}
$$

Obviously, from (III.2) or (III.4), the closed two-form $\omega$ will be non degenerate, and hence symplectic, if $\operatorname{det}(\Omega)=$ $\operatorname{det}(\Psi)=\operatorname{det}(\Phi) \neq 0$, so that $(\Omega)$ has an inverse:

$$
\begin{aligned}
& (\Omega)^{-1}=\left(\begin{array}{cc}
\mathbf{1} & 0 \\
+e \mathbf{F} & \mathbf{1}
\end{array}\right)\left(\begin{array}{cc}
-\Psi^{-1} & 0 \\
0 & \mathbf{1}
\end{array}\right)\left(\begin{array}{cc}
-r \mathbf{G} & \mathbf{1} \\
\mathbf{1} & 0
\end{array}\right) \\
& =\left(\begin{array}{cc}
+\Psi^{-1} r \mathbf{G} & -\Psi^{-1} \\
+e \mathbf{F} \Psi^{-1} r \mathbf{G}+\mathbf{1} & -e \mathbf{F} \Psi^{-1}
\end{array}\right) ; \\
& =\left(\begin{array}{cc}
\mathbf{1} & +r \mathbf{G} \\
0 & \mathbf{1}
\end{array}\right)\left(\begin{array}{cc}
-\mathbf{1} & 0 \\
0 & \Phi^{-1}
\end{array}\right)\left(\begin{array}{cc}
0 & \mathbf{1} \\
\mathbf{1} & -e \mathbf{F}
\end{array}\right) \\
& =\left(\begin{array}{cc}
+r \mathbf{G} \Phi^{-1} & -r \mathbf{G} \Phi^{-1} e \mathbf{F}-\mathbf{1} \\
\Phi^{-1} & -\Phi^{-1} e \mathbf{F}
\end{array}\right) \text {. }
\end{aligned}
$$

Explicitely:

$$
\omega^{b}: \mathbf{d} f \rightarrow\left\{\begin{array}{l}
\left(X_{f}\right)^{i}=\left(\Psi^{-1}\right)_{j}^{i}\left(\partial f / \partial p_{j}-r G^{j k} \partial f / \partial q^{k}\right) \\
\left(X_{f}\right)_{k}=-\left(\Phi^{-1}\right)_{k}{ }^{l}\left(\partial f / \partial q^{l}-e F_{l j} \partial f / \partial p_{j}\right)
\end{array}\right.
$$

The corresponding Poisson brackets are given by:

$$
\{f, g\}=\omega\left(\mathbf{X}_{f}, \mathbf{X}_{g}\right)=\left(\partial_{q} f \partial_{p} f\right)(\Lambda)\left(\begin{array}{c}
\partial_{q} g \\
\partial_{p} g
\end{array}\right)
$$

with the matrix 


$$
(\Lambda)=-(\Omega)^{-1}=\left(\begin{array}{cc}
-\left(\Psi^{-1} r \mathbf{G}=r \mathbf{G} \Phi^{-1}\right) & +\Psi^{-1} \\
-\Phi^{-1} & +\left(\Phi^{-1} e \mathbf{F}=e \mathbf{F} \Psi^{-1}\right)
\end{array}\right)
$$

Explicitely:

$$
\begin{aligned}
\{f, g\}= & -\frac{\partial f}{\partial q}\left(\Psi^{-1} r G\right) \frac{\partial g}{\partial q}-\frac{\partial f}{\partial p}\left(\Phi^{-1}\right) \frac{\partial g}{\partial q} \\
& +\frac{\partial f}{\partial q}\left(\Psi^{-1}\right) \frac{\partial g}{\partial p}+\frac{\partial f}{\partial p}\left(\Phi^{-1} e F\right) \frac{\partial g}{\partial p}
\end{aligned}
$$

In particular, for the coordinates $\left(q^{i}, p_{k}\right)$, we have:

$$
\begin{aligned}
\left\{q^{i}, q^{j}\right\} & =-\left(\Psi^{-1}\right)^{i}{ }_{k} r G^{k j}=-r G^{i k}\left(\Phi^{-1}\right)_{k}{ }^{j}, \\
\left\{q^{i}, p_{l}\right\} & =\left(\Psi^{-1}\right)^{i}{ }_{l}, \\
\left\{p_{k}, q^{j}\right\} & =-\left(\Phi^{-1}\right)_{k}{ }^{j}, \\
\left\{p_{k}, p_{l}\right\} & =\left(\Phi^{-1}\right)_{k}{ }^{j} e F_{j l}=e F_{k j}\left(\Psi^{-1}\right)^{j}{ }_{l} .
\end{aligned}
$$

With $\mathcal{H}(q, p)=\left(\delta^{k l} p_{k} p_{l} / 2 m\right)+\mathcal{V}(q)$, the equations of motion read:

$$
\begin{aligned}
\frac{d q^{i}}{d t} & =\left\{q^{i}, \mathcal{H}\right\}=\left(\Psi^{-1}\right)_{j}^{i}\left(-r G^{j k} \frac{\partial \mathcal{H}}{\partial q^{k}}+\frac{\partial \mathcal{H}}{\partial p_{j}}\right) \\
& =\left(\Psi^{-1}\right)_{j}^{i}\left(-r G^{j k} \frac{\partial \mathcal{V}}{\partial q^{k}}+\frac{p^{j}}{m}\right) \\
\frac{d p_{k}}{d t} & =\left\{p_{k}, \mathcal{H}\right\}=\left(\Phi^{-1}\right)_{k}^{l}\left(-\frac{\partial \mathcal{H}}{\partial q^{l}}+e F_{l j} \frac{\partial \mathcal{H}}{\partial p_{j}}\right) \\
& =\left(\Phi^{-1}\right)_{k}{ }^{l}\left(-\frac{\partial \mathcal{V}}{\partial q^{l}}+e F_{l j} \frac{p^{j}}{m}\right) .
\end{aligned}
$$

The celebrated Darboux theorem guarantees the existence of local coordinates $\left(\xi^{i}, \pi_{k}\right)$, such that $\omega=\mathbf{d} \xi^{i} \wedge \mathbf{d} \pi_{i}$. When one of the charges $(e, r)$ vanishes, such Darboux coordinates are easily obtained using the potential one-forms $\mathbf{A}=A_{i}(q) \mathbf{d} q^{i}$ and $\widetilde{\mathbf{A}}=\widetilde{A}^{k}(p) \mathbf{d} p_{k}$, such that $\mathbf{F}=\mathbf{d A}$ and $\mathbf{G}=\mathbf{d} \widetilde{\mathbf{A}}$.

Indeed, if $r=0$, as in section II, Darboux coordinates are provided by $\xi^{i}=q^{i} ; \pi_{k}=p_{k}+e A_{k}(q)$. A modified symplectic potential and two-form are defined by:

$$
\theta=\left(p_{k}+e A_{k}\right) \mathbf{d} q^{k} ; \omega=-\mathbf{d} \theta .
$$

The Hamiltonian and corresponding equations of motion are:

$$
\begin{gathered}
\mathcal{H}(\xi, \pi)=\frac{1}{2} \delta^{k l}\left(\pi_{k}-e A_{k}(\xi)\right)\left(\pi_{l}-e A_{l}(\xi)\right)+\mathcal{V}(\xi), \text { (III.14) } \\
\frac{\mathbf{d} \xi^{i}}{\mathbf{d} t}=\delta^{i j}\left(\pi_{j}-e A_{j}(\xi)\right), \frac{\mathbf{d} \pi_{i}}{\mathbf{d} t}=e \delta^{k l}\left(\pi_{k}-e A_{k}\right) \frac{\partial A_{l}}{\partial \xi^{i}}-\frac{\partial \mathcal{V}}{\partial \xi^{i}},
\end{gathered}
$$

which yields the second order equation in $\xi$, as in (II.1):

$$
\frac{\mathbf{d}^{2} \xi^{i}}{\mathbf{d} t^{2}}=\delta^{i j}\left(-\frac{\partial \mathcal{V}(\xi)}{\partial \xi^{j}}+e F_{j l}(\xi) \frac{\mathbf{d} \xi^{l}}{\mathbf{d} t}\right)
$$

When $e=0$, Darboux variables are

$$
\xi^{i}=q^{i}+r \widetilde{A}^{i}(p) ; \pi_{k}=p_{k}
$$

and we define

$$
\theta=p_{k} \mathbf{d}\left(q^{k}+r \widetilde{A}^{k}\right) ; \omega=-\mathbf{d} \theta .
$$

The Hamiltonian and equations of motion are now given by:

$$
\begin{gathered}
\mathcal{H}(\xi, \pi)=\frac{1}{2} \delta^{k l} \pi_{k} \pi_{l}+\mathcal{V}(\xi-r \widetilde{A}(\pi)), \\
\frac{\mathbf{d} \xi^{i}}{\mathbf{d} t}=\delta^{i j} \pi_{j}-r \partial_{k} \mathcal{V}(q) \frac{\partial \widetilde{A}^{k}}{\partial \pi_{i}}, \frac{\mathbf{d} \pi_{i}}{\mathbf{d} t}=-\frac{\partial \mathcal{V}}{\partial q^{i}}(q) .
\end{gathered}
$$

The second order equation, obeyed by $\pi(!)$, is given by

$$
\frac{\mathbf{d}^{2} \pi_{i}}{\mathbf{d} t^{2}}=\partial_{i j}^{2} \mathcal{V}(q)\left(-\delta^{j k} \pi_{k}+r G^{j k}(\pi) \frac{\mathbf{d} \pi_{l}}{\mathbf{d} t}\right) .
$$

Here the $q$-variable is assumed to be solved in terms of $\dot{\pi}$ from equation $\dot{\pi}_{k}=-\partial \mathcal{V}(q) / \partial q^{k}$ and this is possible if $\operatorname{det}\left(\partial_{i j}^{2} \mathcal{V}(q)\right) \neq 0$ !

In the case of nonzero charges $(e, r)$ and non-constant $\mathbf{F}$ and $\mathbf{G}$ fields, there is no generic formula to define global Darboux coordinates $\left(\xi^{i}, \pi_{k}\right)$. However, if the fields $\mathbf{F}$ and $\mathbf{G}$ are constant, the Poisson matrix (III.2) is brought in canonical Darboux form by a linear symplectic orthogonalization procedure, à la Hilbert-Schmidt. In the next section this is done explicitely for $N=2$ and $N=3$. Obviously such a linear transformation: $\left(q^{i}, p_{k}\right) \Rightarrow\left(\xi^{i}, \pi_{k}\right)$ is defined up to a linear symplectic map of $\mathbf{S p}(2 n)$. These variables $\left(\xi^{i}, \pi_{k}\right) \in \mathbf{R}^{2 n}$ can be canonically quantised as operators obeying the commutation relations

$$
\left[\widehat{\xi^{i}}, \widehat{\xi^{j}}\right]=0 ;\left[\widehat{\xi^{i}}, \widehat{\pi}_{l}\right]=i \hbar \delta_{l}^{i} ;\left[\widehat{\pi}_{k}, \widehat{\pi}_{l}\right]=0
$$

As von Neumann taught us in [1], they are realised on the Hilbert space of square integrable functions of the variable $\xi$ as

$$
(\widehat{\xi} \Psi)(\xi)=\xi^{i} \Psi(\xi) ;\left(\widehat{\pi_{k}} \Psi\right)(\xi)=\frac{\hbar}{i} \frac{\partial \Psi(\xi)}{\partial \xi^{k}}
$$

The original variables $\left(q^{i}, p_{k}\right)$ being linear functions of the $\left(\xi^{i}, \pi_{k}\right)$ are then also quantised.

When $\operatorname{det}(\Psi)=\operatorname{det}(\Phi)=0$, the closed two-form $\omega$ is singular. When its rank is constant, $\omega$ defines a presymplectic structure on phase space which we call the primary constraint manifold denoted by $\mathcal{M}_{1}$. The consistency of the resulting constrained Hamiltonian system will be examined in the $N=2$ and $N=3$ cases. 


\section{EXAMPLES: $N=2$ AND 3}

In the two examples below, we consider a classical Hamiltonian of the form

$$
\mathcal{H}=\frac{1}{2 m} \delta^{k l} p_{k} p_{l}+\mathcal{V}(q) .
$$

A complete resolution will be given for a harmonic oscillator potential:

$$
\mathcal{V}(q) \doteq \frac{\kappa}{2} \delta_{i j} q^{i} q^{j} .
$$

Also of interest is the case of a constant "electric field": $\mathcal{V}(q)=-\mathbf{E}_{k} q^{k}$, which is exactly solvable and left to the reader.

\section{A. Dynamics in the noncommutative plane}

The magnetic fields in two dimensions, are written as:

$$
e F_{i j}=B \varepsilon_{i j} ; r G^{k l}=C \varepsilon^{k l},
$$

where $B$ and $C$ are pseudoscalars. The closed two-form (III.1) becomes

$$
\omega=\mathbf{d} q^{i} \wedge \mathbf{d} p_{i}-B \mathbf{d} q^{1} \wedge \mathbf{d} q^{2}+C \mathbf{d} p_{1} \wedge \mathbf{d} p_{2} .
$$

The equation $\iota_{X} \omega=\mathbf{d} f$ reads

$$
X^{i}-C \varepsilon^{i j} X_{j}=\frac{\partial f}{\partial p_{i}} ; X_{k}-B \varepsilon_{k l} X^{l}=-\frac{\partial f}{\partial q^{k}} .
$$

Denoting $\chi \doteq(1+C B)$, the matrices $\Phi$ and $\Psi$ are written as $\Phi_{i}{ }^{j}=\chi \delta_{i}{ }^{j}$ and $\Psi^{k}{ }_{l}=\chi \delta^{k}{ }_{l}$. The matrix (III.2) is then invertible if $\chi$ does not vanish.

\section{The non degenerate case}

Here, we will assume $\chi$ to be strictly positive. The above equation (IV.5) can then be inverted with Hamiltonian vector fields given by:

$X^{i}=\chi^{-1}\left(\frac{\partial f}{\partial p_{i}}-C \varepsilon^{i j} \frac{\partial f}{\partial q^{j}}\right), X_{k}=-\chi^{-1}\left(\frac{\partial f}{\partial q^{k}}-B \varepsilon_{k l} \frac{\partial f}{\partial p^{l}}\right)$

The Poisson brackets (III.11) become:

$$
\begin{aligned}
& \left\{q^{i}, q^{j}\right\}=-C \chi^{-1} \varepsilon^{i j} ;\left\{q^{i}, p_{l}\right\}=\chi^{-1} \delta^{i} l \\
& \left\{p_{k}, q^{j}\right\}=-\chi^{-1} \delta_{k}^{j} ;\left\{p_{k}, p_{l}\right\}=B \chi^{-1} \varepsilon_{k l} .
\end{aligned}
$$

Substitution of the Ansatz

$$
\xi^{i}=\alpha q^{i}+\beta \frac{C}{2} p_{k} \varepsilon^{k i} ; \pi_{k}=\gamma \frac{B}{2} q^{j} \varepsilon_{j k}+\delta p_{k},
$$

in the canonical Poison brackets, leads to the equations

$$
\begin{aligned}
& \alpha^{2}-\alpha \beta-\frac{C B}{4} \beta^{2}=0, \delta^{2}-\delta \gamma-\frac{C B}{4} \gamma^{2}=0, \\
& \alpha \delta+\frac{C B}{2}(\alpha \gamma+\delta \beta)-\frac{C B}{4} \beta \gamma=\chi .
\end{aligned}
$$

We choose the solution:

$$
\alpha=\delta=\sqrt{u} ; \beta=\gamma=\frac{1}{\sqrt{u}} ; u=\frac{1}{2}(1+\sqrt{\chi}),
$$

such that (IV.8) reduces to (II.9) when $C=0$ or to (III.17) in case $B=0$. The 2-form (III.1) has the canonical Darboux form $\omega=d \xi^{i} \wedge d \pi_{i}$ in the variables

$$
\xi^{i}=\sqrt{u}\left(q^{i}-\frac{C}{2 u} \varepsilon^{i k} p_{k}\right) ; \pi_{k}=\sqrt{u}\left(p_{k}-\frac{B}{2 u} \varepsilon_{k i} q^{i}\right) .
$$

These have an inverse if, and only if $\chi \neq 0$ :

$$
\sqrt{\chi} q^{i}=\sqrt{u}\left(\xi^{i}+\frac{C}{2 u} \varepsilon^{i k} \pi_{k}\right) ; \sqrt{\chi} p_{k}=\sqrt{u}\left(\pi_{k}+\frac{B}{2 u} \varepsilon_{k i} \xi^{i}\right) .
$$

With the complex variables

$$
q=q^{1}+\mathbf{i} q^{2}, p=p_{1}+\mathbf{i} p_{2} ; \xi=\xi^{1}+\mathbf{i} \xi^{2}, \pi=\pi_{1}+\mathbf{i} \pi_{2},
$$

the above changes of variables are written as:

$$
\xi=\sqrt{u}\left(q+\mathbf{i} \frac{C}{2 u} p\right) ; \pi=\sqrt{u}\left(p+\mathbf{i} \frac{B}{2 u} q\right) .
$$

The inverse transformations are:

$$
q=\sqrt{u / \chi}\left(\xi-\mathbf{i} \frac{C}{2 u} \pi\right) ; p=\sqrt{u / \chi}\left(\pi-\mathbf{i} \frac{B}{2 u} \xi\right) .
$$

The Hamiltonian (IV.2) becomes

$$
\begin{aligned}
\mathcal{H} & =\frac{1}{2 m^{\prime}} \delta^{k l} \pi_{k} \pi_{l}+\frac{\kappa^{\prime}}{2} \delta_{i j} \xi^{i} \xi^{j}-\omega_{L}^{\prime} \Lambda \\
& =\frac{1}{2 m^{\prime}} \frac{\pi^{\dagger} \pi+\pi \pi^{\dagger}}{2}+\frac{\kappa^{\prime}}{2} \frac{\xi^{\dagger} \xi+\xi \xi^{\dagger}}{2}-\omega_{L}^{\prime} \Lambda
\end{aligned}
$$

where $\Lambda$ is angular momentum

$$
\begin{aligned}
\Lambda & =\frac{1}{2}\left(\varepsilon_{i j} \xi^{i} \delta^{j k} \pi_{k}-\varepsilon^{k l} \pi_{k} \delta_{l j} \xi^{j}\right) \\
& =\frac{1}{2}\left(\left(\xi^{1} \pi_{2}-\xi^{2} \pi_{1}\right)-\left(\pi_{1} \xi^{2}+\pi_{2} \xi^{1}\right)\right) \\
& =\frac{1}{4 \mathbf{i}}\left(\left(\xi^{\dagger} \pi-\xi \pi^{\dagger}\right)-\left(\pi \xi^{\dagger}+\pi^{\dagger} \xi\right)\right) .
\end{aligned}
$$

The "renormalised" mass and elasticity constant are given by:

$$
\frac{1}{m^{\prime}}=\frac{1}{m} \frac{u}{\chi}\left(1+\frac{c^{2}}{4 u^{2}}\right) ; \kappa^{\prime}=\kappa \frac{u}{\chi}\left(1+\frac{b^{2}}{4 u^{2}}\right) .
$$

where

$$
b=\frac{B}{\sqrt{m \kappa}} ; c=C \sqrt{m \kappa} .
$$

The corresponding frequency $\omega_{0}^{\prime}=\sqrt{\kappa^{\prime} / m^{\prime}}$ is given in terms of the "bare" frequency $\omega_{0}=\sqrt{\kappa / m}$ by:

$$
\omega_{0}^{\prime}=\frac{\omega_{0}}{2 \chi}\left((b-c)^{2}+4 \chi\right)^{1 / 2} \text {. }
$$


and $\omega_{L}^{\prime}$, the induced Larmor frequency, by:

$$
\omega_{L}^{\prime}=\frac{\omega_{0}}{2 \chi}(b-c) .
$$

The solution of Hamiltonian's equations with (IV.16) is standard. With[22]

$$
m^{\prime} \omega_{0}^{\prime}=\sqrt{m^{\prime} \kappa^{\prime}}=\sqrt{m \kappa}\left(\left(1+\frac{b^{2}}{4 u^{2}}\right)\left(1+\frac{c^{2}}{4 u^{2}}\right)^{-1}\right)^{1 / 2}
$$

reduced variables are introduced by:

$$
Q \doteq\left(m^{\prime} \omega_{0}^{\prime}\right)^{1 / 2} \xi ; P \doteq\left(m^{\prime} \omega_{0}^{\prime}\right)^{-1 / 2} \pi .
$$

The original $(q, p)$ are expressed as:

$$
\begin{aligned}
& q=\sqrt{u / \chi}\left(m^{\prime} \omega_{0}^{\prime}\right)^{-1 / 2}\left(Q-\mathbf{i} \frac{c^{\prime}}{2 u} P\right), \\
& p=\sqrt{u / \chi}\left(m^{\prime} \omega_{0}^{\prime}\right)^{+1 / 2}\left(P-\mathbf{i} \frac{b^{\prime}}{2 u} Q\right),
\end{aligned}
$$

where

$$
c^{\prime}=C\left(m^{\prime} \omega_{0}^{\prime}\right)=C \sqrt{m^{\prime} \kappa^{\prime}}, b^{\prime}=B /\left(m^{\prime} \omega_{0}^{\prime}\right)=B / \sqrt{m^{\prime} \kappa^{\prime}} .
$$

The symplectic structure and the Poisson brackets are:

$$
\begin{aligned}
\omega & =\frac{1}{2}\left(\mathbf{d} Q^{\dagger} \wedge \mathbf{d} P+\mathbf{d} Q \wedge \mathbf{d} P^{\dagger}\right) \\
\{f, g\} & =2\left(\frac{\partial f}{\partial Q} \frac{\partial g}{\partial P^{\dagger}}+\frac{\partial f}{\partial Q^{\dagger}} \frac{\partial g}{\partial P}-\frac{\partial f}{\partial P} \frac{\partial g}{\partial Q^{\dagger}}-\frac{\partial f}{\partial P^{\dagger}} \frac{\partial g}{\partial Q}\right) .
\end{aligned}
$$

The fundamental nonzero Poisson bracket is

$$
\left\{Q, P^{\dagger}\right\}=2 .
$$

In these variables, the Hamiltonian (IV.16) reads:

$$
\mathcal{H}=\frac{\omega_{0}^{\prime}}{4}\left(\left(P^{\dagger} P+P P^{\dagger}\right)+\left(Q^{\dagger} Q+Q Q^{\dagger}\right)\right)-\omega_{L}^{\prime} \Lambda, \quad(\mathrm{IV} .28)
$$

where

$$
\Lambda=\frac{1}{4 \mathbf{i}}\left(\left(Q^{\dagger} P-Q P^{\dagger}\right)-\left(P Q^{\dagger}+P^{\dagger} Q\right)\right) .
$$

The corresponding equations of motion are:

$$
\begin{aligned}
& \frac{d Q}{d t}=\{Q, \mathcal{H}\}=2 \frac{\partial \mathcal{H}}{\partial P^{\dagger}}=\omega_{0}^{\prime} P-\mathbf{i} \omega_{L}^{\prime} Q \\
& \frac{d P}{d t}=\{Q, \mathcal{H}\}=-2 \frac{\partial \mathcal{H}}{\partial Q^{\dagger}}=-\omega_{0}^{\prime} Q-\mathbf{i} \omega_{L}^{\prime} P(\text { IV.30) }
\end{aligned}
$$

With the shift variables

$$
A_{(+)}=\frac{1}{2}(Q+\mathbf{i} P) ; A_{(-)}=\frac{1}{2}\left(Q^{\dagger}+\mathbf{i} P^{\dagger}\right),
$$

the symplectic structure and the Poisson brackets are given by:

$$
\mathbf{\omega}=-\mathbf{i}\left(\mathbf{d} A_{(+)}^{\dagger} \wedge \mathbf{d} A_{(+)}+\mathbf{d} A_{(-)}^{\dagger} \wedge \mathbf{d} A_{(-)}\right),
$$

$$
\begin{aligned}
\{f, g\}=-\mathbf{i}( & \frac{\partial f}{\partial A_{(+)}} \frac{\partial g}{\partial A_{(+)}^{\dagger}}+\frac{\partial f}{\partial A_{(-)}} \frac{\partial g}{\partial A_{(-)}^{\dagger}} \\
& \left.-\frac{\partial f}{\partial A_{(+)}^{\dagger}} \frac{\partial g}{\partial A_{(+)}}-\frac{\partial f}{\partial A_{(-)}^{\dagger}} \frac{\partial g}{\partial A_{(-)}}\right)
\end{aligned}
$$

with fundamental nonzero brackets:

$$
\left\{A_{( \pm)}, A_{( \pm)}^{\dagger}\right\}=-\mathbf{i}
$$

The Hamiltonian, with the (positive !) frequencies

$$
\omega_{( \pm)}=\left(\omega_{0}^{\prime} \pm \omega_{L}^{\prime}\right)
$$

reads now:

$$
\mathcal{H}=\frac{\omega_{(+)}}{2}\left(A_{(+)}^{\dagger} A_{(+)}+A_{(+)} A_{(+)}^{\dagger}\right)+\frac{\omega_{(-)}}{2}\left(A_{(-)}^{\dagger} A_{(-)}+A_{(-)} A_{(-)}^{\dagger}\right) .
$$

The corresponding equations of motion and their solutions are given by:

$$
\begin{gathered}
\frac{d A_{( \pm)}}{d t}=\left\{A_{( \pm)}, \mathcal{H}\right\}=-\mathbf{i} \frac{\partial \mathcal{H}}{\partial A_{( \pm)}^{\dagger}}=-\mathbf{i} \omega_{( \pm)} A_{( \pm)} \\
A_{( \pm)}(t)=\exp \left\{-\mathbf{i} \omega_{( \pm)} t\right\} A_{( \pm)}(0) .
\end{gathered}
$$


The relations between variables are given by:

$$
\begin{aligned}
A_{(+)} & =\frac{1}{2}(Q+\mathbf{i} P) \\
& =\frac{\sqrt{u}}{2}\left(\left(m^{\prime} \omega_{0}^{\prime}\right)^{+1 / 2}\left(1-\frac{b^{\prime}}{2 u}\right) q+\mathbf{i}\left(m^{\prime} \omega_{0}^{\prime}\right)^{-1 / 2}\left(1+\frac{c^{\prime}}{2 u}\right) p\right) \\
A_{(-)}^{\dagger} & =\frac{1}{2}(Q-\mathbf{i} P) \\
& =\frac{\sqrt{u}}{2}\left(\left(m^{\prime} \omega_{0}^{\prime}\right)^{+1 / 2}\left(1+\frac{b^{\prime}}{2 u}\right) q-\mathbf{i}\left(m^{\prime} \omega_{0}^{\prime}\right)^{-1 / 2}\left(1-\frac{c^{\prime}}{2 u}\right) p\right) .
\end{aligned}
$$

The inverse transformations are:

$$
\begin{aligned}
q & =\left(m^{\prime} \omega_{0}^{\prime}\right)^{-1 / 2} \sqrt{u / \chi}\left(Q-\mathbf{i} \frac{c^{\prime}}{2 u} P\right) \\
& =\left(m^{\prime} \omega_{0}^{\prime}\right)^{-1 / 2} \sqrt{u / \chi}\left(\left(1-\frac{c^{\prime}}{2 u}\right) A_{(+)}+\left(1+\frac{c^{\prime}}{2 u}\right) A_{(-)}^{\dagger}\right), \\
p & =\left(m^{\prime} \omega_{0}^{\prime}\right)^{+1 / 2} \sqrt{u / \chi}\left(P-\mathbf{i} \frac{b^{\prime}}{2 u} Q\right) \\
& =\mathbf{i}\left(m^{\prime} \omega_{0}^{\prime}\right)^{+1 / 2} \sqrt{u / \chi}\left(\left(1-\frac{b^{\prime}}{2 u}\right) A_{(-)}^{\dagger}-\left(1+\frac{b^{\prime}}{2 u}\right) A_{(+)}\right) .
\end{aligned}
$$

Quantisation is trivial though the substitution of the fundamental Poison brackets (IV.27),(IV.34) by operator commutators

$$
\left[\mathbf{Q}, \mathbf{P}^{\dagger}\right]=2 \mathbf{i} \hbar ;\left[\mathbf{A}_{( \pm)}, \mathbf{A}_{( \pm)}^{\dagger}\right]=\hbar \text {. }
$$

Having kept the initial ordering, the quantum Hamiltonian has eigenvalues:

$$
E\left(n_{(+)}, n_{(-)}\right)=\hbar \omega_{(+)}\left(n_{(+)}+1 / 2\right)+\hbar \omega_{(-)}\left(n_{(-)}+1 / 2\right),
$$

where $n_{( \pm)}$are nonnegative integers. The corresponding eigenvectors are denoted by $\mid n_{(+)}, n_{(-)}>$.

\section{The degenerate or constraint case}

The condition $\chi \doteq(1+B C)=0$ determines $\omega$ as a presymplectic structure on $\mathcal{M}$ and shall be called the primary constraint. Again, the notation is simplified using complex variables[23]. The presymplectic two-form reads

$$
\begin{aligned}
\omega= & \frac{1}{2}\left(d q^{\dagger} \wedge d p+d q \wedge d p^{\dagger}\right) \\
& -\frac{B}{4 \mathbf{i}}\left(d q^{\dagger} \wedge d q-d q \wedge d q^{\dagger}\right)+\frac{C}{4 \mathbf{i}}\left(d p^{\dagger} \wedge d p-d p \wedge d p^{\dagger}\right) .
\end{aligned}
$$

The Hamiltonian (IV.2) becomes

$$
\mathcal{H}=\frac{1}{2 m} \frac{p^{\dagger} p+p p^{\dagger}}{2}+\frac{\kappa}{2} \frac{q^{\dagger} q+q q^{\dagger}}{2}
$$

Writing a vector field as

$$
\begin{gathered}
\mathbf{X}=X^{i} \partial / \partial q^{i}+X_{k} \partial / \partial p_{k}=U \partial / \partial q+U^{\dagger} \partial / \partial q^{\dagger}+V \partial / \partial p+V^{\dagger} \partial / \partial p^{\dagger} \\
l_{X} \omega=\frac{1}{2}\left((U+\mathbf{i} C V) d q^{\dagger}+\left(U^{\dagger}-\mathbf{i} C V^{\dagger}\right) d q\right. \\
\left.-(V+\mathbf{i} B U) d p^{\dagger}-\left(V^{\dagger}-\mathbf{i} B U^{\dagger}\right) d p\right) .
\end{gathered}
$$

The homogeneous equation, $\iota_{\mathbf{Z}} \omega=0$ has nontrivial solutions. Indeed, with $U_{0}=Z^{1}+\mathbf{i} Z^{2}$ and $V_{0}=Z_{1}+\mathbf{i} Z_{2}$, equation
(IV.45) yields the system:

$$
U_{0}+\mathbf{i} C V_{0}=0 ; \text { or } \quad V_{0}+\mathbf{i} B U_{0}=0,
$$


of which the determinant is $\chi=1+B C=0$.

The inhomogeneous equation $l_{\mathbf{X}} \boldsymbol{\omega}=\mathbf{d} \mathcal{H}$, i.e. the Hamiltonian dynamics, reads

$$
U+\mathbf{i} C V=2 \frac{\partial \mathcal{H}}{\partial p^{\dagger}}=\frac{p}{m} ; V+\mathbf{i} B U=-2 \frac{\partial \mathcal{H}}{\partial q^{\dagger}}=\kappa q
$$

It will have a solution if

$$
\langle\mathbf{d} \mathcal{H} \mid \mathbf{Z}\rangle=0 \text {. }
$$

This condition, termed secondary constraint, is explicitely given by:

$$
\frac{\partial \mathcal{H}}{\partial p}-\mathbf{i} C \frac{\partial \mathcal{H}}{\partial q}=0 ; \text { or } \quad \frac{\partial \mathcal{H}}{\partial q}-\mathbf{i} B \frac{\partial \mathcal{H}}{\partial p}=0 .
$$

For the Hamiltonian (IV.44) this condition (IV.49) is linear:

$$
\frac{1}{m} p+\mathbf{i} C \kappa q=0 ; \text { or } \quad \kappa q+\mathbf{i} B \frac{1}{m} p=0 .
$$

and defines the secondary constraint manifold $\mathcal{M}_{2}$.

On $\mathcal{M}_{2}$, a particular solution of $l_{\mathbf{X}} \omega=\mathbf{d} \mathcal{H}$ is given by:

$$
U_{P}=\frac{p}{m} ; V_{P}=0
$$

The general solution is given by:

$$
U=\frac{p}{m}+U_{0} ; V=V_{0} .
$$

where $\left(U_{0}, V_{0}\right)$ is restricted to obey (IV.46). This vector field, restricted to $\mathcal{M}_{2}$, should conserve the constraints i.e. must be tangent to $\mathcal{M}_{2}$ :

$$
0=\left\langle\frac{1}{m} \mathbf{d} p+\mathbf{i} C \kappa \mathbf{d} q \mid X\right\rangle
$$

The vector fields $U$ and $V$ are completely defined on $\mathcal{M}_{2}$, with ensuing equations of motion:

$$
\begin{aligned}
& \frac{d q}{d t}=U=-\mathbf{i} \frac{\sqrt{m \kappa} C}{1+m \kappa C^{2}} \omega_{0} q=\frac{1}{1+m \kappa C^{2}} \frac{p}{m} \\
& \frac{d p}{d t}=V=-\mathbf{i} \frac{\sqrt{m \kappa} C}{1+m \kappa C^{2}} \omega_{0} p=-\frac{m \kappa C^{2}}{1+m \kappa C^{2}} \kappa q .
\end{aligned}
$$

In terms of the frequency:

$$
\omega_{r}=-\frac{\sqrt{m \kappa} C}{1+m \kappa C^{2}} \omega_{0}=\frac{B / \sqrt{m \kappa}}{1+B^{2} / m \kappa} \omega_{0},
$$

the solution is given by

$$
q(t)=\exp \left\{\mathbf{i} \omega_{r} t\right\} q_{0} ; p(t)=\exp \left\{\mathbf{i} \omega_{r} t\right\} p_{0} .
$$

Obviously, if $q_{0}$ and $p_{0}$ obey the secondary constraints (IV.50), $q(t)$ and $p(t)$ obey them at all times.

The same result can be obtained by symplectic reduction, restricting the pre-symplectic two-form (IV.43) to $\mathcal{M}_{2}$ :

$$
\begin{gathered}
\omega_{\mid \mathcal{M}_{2}}=-\mathbf{i} \frac{\left(1+m \kappa C^{2}\right)^{2}}{2 C} d q^{\dagger} \wedge d q . \\
\{f, g\}_{\mathcal{M}_{2}}=\frac{2 \mathbf{i} C}{\left(1+m \kappa C^{2}\right)^{2}}\left(\frac{\partial f}{\partial q^{\dagger}} \frac{\partial g}{\partial q}-\frac{\partial f}{\partial q} \frac{\partial g}{\partial q^{\dagger}}\right) .
\end{gathered}
$$

The fundamental Poisson bracket is

$$
\left\{q, q^{\dagger}\right\}_{\mathscr{M}_{2}}=\frac{-2 \mathbf{i} C}{\left(1+m \kappa C^{2}\right)^{2}}
$$

The dynamics are given by:

$$
\frac{d q}{d t}=-\frac{2 \mathbf{i} C}{(1+m \kappa C)^{2}} \frac{\partial \mathcal{H}_{r}}{\partial q^{\dagger}} .
$$

And, with the reduced Hamiltonian $\mathcal{H}_{r}$ given by

$$
\mathcal{H}_{r}=\left(1+m \kappa C^{2}\right) \frac{\kappa}{2} q^{\dagger} q,
$$

this yields equation (IV.56). When $B>0$, hence $C<0$, we define

$$
a=\frac{\left(1+m \kappa C^{2}\right)}{|2 C|} q^{\dagger}
$$

such that

$$
\left\{a, a^{\dagger}\right\}=-\mathbf{i} ; \mathcal{H}_{r}=\frac{\omega_{r}}{2}\left(a^{\dagger} a+a a^{\dagger}\right) .
$$

Quantisation is again trivial introducing operators $\mathbf{a}$ and $\mathbf{a}^{\dagger}$, obeying

$$
\left[\mathbf{a}, \mathbf{a}^{\dagger}\right]=\hbar
$$

such that the quantum Hamiltonian

$$
\mathbf{H}_{r}=\frac{\omega_{r}}{2}\left(\mathbf{a}^{\dagger} \mathbf{a}+\mathbf{a} \mathbf{a}^{\dagger}\right)
$$

has eigenvalues:

$$
E(n)=\hbar \omega_{r}(n+1 / 2)
$$




\section{The $\chi \rightarrow 0$ limit of (IV A 1).}

We need the expansion of

$$
\left(m^{\prime} \omega_{0}^{\prime}\right)=\left(m \omega_{0}\right) \times\left(\left(1+\frac{b^{2}}{4 u^{2}}\right)\left(1+\frac{c^{2}}{4 u^{2}}\right)^{-1}\right)^{1 / 2}
$$

in powers of $\varepsilon=\sqrt{\chi}$, where $1+b c=\varepsilon^{2}$ and $2 u=1+\varepsilon$.

$$
\begin{aligned}
\left(m^{\prime} \omega_{0}^{\prime}\right) & =\frac{m \omega_{0}}{|c|}\left(1+\frac{c^{2}-1}{c^{2}+1} \varepsilon+\cdots\right) \\
& =\frac{1}{|C|}\left(1+\frac{c^{2}-1}{c^{2}+1} \varepsilon+\cdots\right) \\
\left(m^{\prime} \omega_{0}^{\prime}\right)^{-1} & =\frac{\left(m \omega_{0}\right)^{-1}}{|b|}\left(1+\frac{b^{2}-1}{b^{2}+1} \varepsilon+\cdots\right) \\
& =\frac{1}{|B|}\left(1+\frac{b^{2}-1}{b^{2}+1} \varepsilon+\cdots\right) .
\end{aligned}
$$

Also, from (IV.25), we obtain

$$
\begin{aligned}
& \frac{c^{\prime}}{2 u}=\frac{\left(m^{\prime} \omega_{0}^{\prime}\right) C}{2 u}=\frac{C}{|C|}\left(1-\frac{2}{c^{2}+1} \varepsilon+\cdots,\right) \\
& \frac{b^{\prime}}{2 u}=\frac{B}{\left(m^{\prime} \omega_{0}^{\prime}\right) 2 u}=\frac{B}{|B|}\left(1-\frac{2}{b^{2}+1} \varepsilon+\cdots,\right)(\text { IV.69) }
\end{aligned}
$$

For definitenees, we assume in the following $B>0$ and so $C<0$ in the limit $\varepsilon \rightarrow 0$. We obtain

$$
\begin{gathered}
1-\frac{b^{\prime}}{2 u}=\frac{2}{1+b^{2}} \varepsilon+\cdots ; \\
1+\frac{b^{\prime}}{2 u}=2-\frac{2}{1+b^{2}} \varepsilon+\cdots \\
1+\frac{c^{\prime}}{2 u}=\frac{2}{1+c^{2}} \varepsilon+\cdots ; \\
1-\frac{c^{\prime}}{2 u}=2-\frac{2}{1+b^{2}} \varepsilon+\cdots
\end{gathered}
$$

Also:

$$
\omega_{0}^{\prime}=\frac{\omega_{0}}{2 \varepsilon^{2}}(b-c)\left(1+\frac{2 \varepsilon^{2}}{(b-c)^{2}}\right), \omega_{L}^{\prime}=\frac{\omega_{0}}{2 \varepsilon^{2}}(b-c),
$$

$$
\begin{aligned}
& \omega_{(+)}=\omega_{0}^{\prime}+\omega_{L}^{\prime}=-\omega_{0} \frac{1+\left(m \omega_{0}\right)^{2} C^{2}}{\left(m \omega_{0}\right) C} \frac{1}{\varepsilon^{2}}, \\
& \omega_{(-)}=\omega_{0}^{\prime}-\omega_{L}^{\prime}=-\omega_{0} \frac{\left(m \omega_{0}\right) C}{1+\left(m \omega_{0}\right)^{2} C^{2}} .
\end{aligned}
$$

One of the frequencies $\omega_{(+)}$diverges, while the other $\omega_{(-)}$ tends to $\omega_{r}$ defined in (IV.55). The relations in (IV.39) yield the initial conditions:

$$
\begin{aligned}
& A_{(+)}(0) \approx \sqrt{\frac{|B|}{2}}\left(1+b^{2}\right)^{-1}\left(q_{0}+\mathbf{i} \frac{B}{\left(m \omega_{0}\right)^{2}} p_{0}\right)\left(\varepsilon+O\left(\varepsilon^{2}\right)\right) \\
& A_{(-)}^{\dagger}(0) \approx \sqrt{\frac{|B|}{2}}\left(q_{0}-\mathbf{i} \frac{1}{|B|} p_{0}\right)\left(1+O\left(\varepsilon^{2}\right)\right) .
\end{aligned}
$$

The solutions (IV.40), in the $\varepsilon \rightarrow 0$ limit are then written as

$$
\begin{aligned}
q(t) \approx & \sqrt{\frac{2}{|B|}}\left(\frac{1}{\varepsilon} A_{(+)}(0) \exp \left\{-\mathbf{i} \omega_{(+)} t\right\}+\frac{1}{1+c^{2}} A_{(-)}^{\dagger}(0) \exp \left\{\mathbf{i} \omega_{r} t\right\}\right) \\
\approx & \left(1+b^{2}\right)^{-1}\left(q_{0}+\mathbf{i} \frac{|B|}{\left(m \omega_{0}\right)^{2}} p_{0}\right) \exp \left\{-\mathbf{i} \omega_{(+)} t\right\} \\
& \left.+\left(1+c^{2}\right)^{-1}\left(q_{0}-\mathbf{i}|B|^{-1} p\right)\right) \exp \left\{+\mathbf{i} \omega_{r} t\right\}
\end{aligned}
$$

The first term is a fast oscillating function with diverging frequency and so averages to zero. Furthermore, if the initial conditions are on $\mathcal{M}_{2}$, i.e. if $\left(q_{0}+\mathbf{i}|B| p_{0} /\left(m \omega_{0}\right)^{2}\right)=0$, this first term behaves as $O(\varepsilon) \exp \left\{\mathbf{i} v t / \varepsilon^{2}\right\}$ converging to zero. The second term is then reduced to the expression (IV.56) of $q(t)$. Similar considerations hold for $p(t)$ in such a way that the solution stays on $M_{2}$. 


\section{B. Noncommutative $\mathbf{R}^{3}$}

In $\mathbf{R}^{\mathbf{3}}$, the magnetic fields $\mathbf{F}$ and $\mathbf{G}$ are written in terms of pseudovectors $\overline{\mathbf{B}}=\left\{B^{k}\right\}$ and $\underline{\mathbf{C}}=\left\{C_{k}\right\}$ as:

$$
e F_{i j}=\varepsilon_{i j k} B^{k} ; r G^{i j}=\varepsilon^{i j k} C_{k} .
$$

The closed two-form (III.1) is written as:

$$
\omega=\mathbf{d} q^{i} \wedge \mathbf{d} p_{i}-\frac{1}{2} \varepsilon_{i j k} B^{k} \mathbf{d} q^{i} \wedge \mathbf{d} q^{j}+\frac{1}{2} \varepsilon^{k l m} C_{m} \mathbf{d} p_{k} \wedge \mathbf{d} p_{l} .
$$

The fundamental equation $l_{X} \omega=\mathbf{d} f$ reads

$$
X^{i}-C_{k} \varepsilon^{i j k} X_{j}=\frac{\partial f}{\partial p_{i}} ; X_{k}-B^{i} \varepsilon_{k l i} X^{l}=-\frac{\partial f}{\partial q^{k}} .
$$

Defining $\vartheta=\underline{\mathbf{C}} \cdot \overline{\mathbf{B}}=C_{k} B^{k}$ and $\chi=1+\vartheta$, this is also written as

$$
\begin{aligned}
& \chi X^{i}=\left(\delta_{j}^{i}+B^{i} C_{j}\right) \frac{\partial f}{\partial p_{j}}-C_{k} \varepsilon^{i j k} \frac{\partial f}{\partial q^{j}} \\
& \chi X_{k}=-\left(\left(\delta_{k}{ }^{l}+C_{k} B^{l}\right) \frac{\partial f}{\partial q^{l}}-B^{i} \varepsilon_{k l i} \frac{\partial f}{\partial p_{l}}\right)
\end{aligned}
$$

The $3 \times 3$ matrices $\Phi$ and $\Psi$ read:

$$
\Phi_{i}{ }^{j}=\chi \delta_{i}{ }^{j}-C_{i} B^{j} ; \Psi^{k}{ }_{l}=\chi \delta^{k}{ }_{l}-B^{k} C_{l},
$$

with $\operatorname{det} \Phi=\operatorname{det} \Psi=\chi^{2}$. Assuming again $\chi \neq 0[24]$, these matrices have inverses:

$$
\left(\Phi^{-1}\right)_{i}^{j}=\frac{1}{\chi}\left(\delta_{i}^{j}+C_{i} B^{j}\right),\left(\Psi^{-1}\right)_{\ell}^{k}=\frac{1}{\chi}\left(\delta^{k}+B^{k} C_{\ell}\right)
$$

The Hamiltonian vector fields are obtained from (IV.78):

$$
\begin{aligned}
& X^{i}=\chi^{-1}\left(\left(\delta_{j}^{i}+B^{i} C_{j}\right) \frac{\partial f}{\partial p_{j}}-C_{k} \varepsilon^{i j k} \frac{\partial f}{\partial q^{j}}\right) \\
& X_{k}=-\chi^{-1}\left(\left(\delta_{k}{ }^{l}+C_{k} B^{l}\right) \frac{\partial f}{\partial q^{l}}-B^{i} \varepsilon_{k l i} \frac{\partial f}{\partial p_{l}}\right)
\end{aligned}
$$

The Poissson brackets are given by:

$$
\begin{aligned}
\left\{q^{i}, q^{j}\right\}=-\chi^{-1} \varepsilon^{i j k} C_{k} \quad, & \left\{q^{i}, p_{l}\right\}=\chi^{-1}\left(\delta^{i}+B^{i} C_{l}\right), \\
\left\{p_{k}, q^{j}\right\}=-\chi^{-1}\left(\delta_{k}{ }^{j}+C_{k} B^{j}\right) & , \quad\left\{p_{k}, p_{l}\right\}=\chi^{-1} \varepsilon_{k l m} B^{m} .
\end{aligned}
$$

The Ansatz (IV.8) has to be generalised to

$$
\begin{aligned}
& \xi^{i}=\alpha q^{i}+\alpha^{\prime} B^{i}\left(C_{k} q^{k}\right)-\beta \frac{1}{2} \varepsilon^{i j k} p_{j} C_{k} \\
& \pi_{k}=\alpha p_{k}+\alpha^{\prime}\left(p_{i} B^{i}\right) C_{k}+\beta \frac{1}{2} \varepsilon_{k l m} B^{l} q^{m} .
\end{aligned}
$$

For $\alpha, \beta$ similar equations as in (IV.9) are obtained:

$$
\alpha^{2}-\alpha \beta-\frac{\vartheta}{4} \beta^{2}=0, \alpha^{2}+\vartheta(\alpha \beta)-\frac{\vartheta}{4} \beta^{2}=\chi,
$$

with a the same solution ( $\chi$ assumed to be strictly positive):

$$
\alpha=\sqrt{u} ; \beta=\frac{1}{\sqrt{u}} ; u=\frac{1}{2}(1+\sqrt{\chi}) .
$$

Furthermore, there is an additional equation for $\alpha^{\prime}$ :

$$
\chi\left(\vartheta \alpha^{\prime 2}+2 \alpha \alpha^{\prime}\right)+\left(\alpha^{2}-\alpha \beta+\frac{1}{4} \beta^{2}\right)=0 .
$$

Substituting (IV.83), one obtains

$$
\vartheta \alpha^{\prime 2}+2 \sqrt{u} \alpha^{\prime}+\frac{1}{4 u}=0
$$

with solution, remaining finite when $\vartheta \rightarrow 0$,

$$
\alpha^{\prime}=\sqrt{u} \gamma=\frac{(1-\sqrt{u})}{\vartheta}
$$

The formulae (IV.81) are finally written as:

$$
\begin{aligned}
\xi^{i} & =\sqrt{u}\left(q^{i}+\gamma B^{i}\left(C_{k} q^{k}\right)-\frac{1}{2 u} \varepsilon^{i j k} p_{j} C_{k}\right) ; \\
\pi_{k} & =\sqrt{u}\left(p_{k}+\gamma\left(p_{i} B^{i}\right) C_{k}+\frac{1}{2 u} \varepsilon_{k l m} B^{l} q^{m}\right)
\end{aligned}
$$

In old fashioned vector notation, this appears as:

$$
\begin{aligned}
& \bar{\xi}=\sqrt{u}\left(\overline{\mathbf{q}}+\gamma \overline{\mathbf{B}}(\underline{\mathbf{C}} \cdot \overline{\mathbf{q}})-\frac{1}{2 u} \underline{\mathbf{p}} \times \underline{\mathbf{C}}\right) ; \\
& \underline{\pi}=\sqrt{u}\left(\underline{\mathbf{p}}+\gamma(\underline{\mathbf{p}} \cdot \overline{\mathbf{B}}) \underline{\mathbf{C}}+\frac{1}{2 u} \overline{\mathbf{B}} \times \overline{\mathbf{q}}\right) .
\end{aligned}
$$

The inverse formulae of (IV.86) are obtained as:

$$
\begin{aligned}
q^{i} & =\frac{\sqrt{u}}{\sqrt{\chi}}\left(\xi^{i}+\gamma^{\prime} B^{i}\left(C_{k} \xi^{k}\right)+\frac{1}{2 u} \varepsilon^{i j k} \pi_{j} C_{k}\right) \\
p_{k} & =\frac{\sqrt{u}}{\sqrt{\chi}}\left(\pi_{k}+\gamma^{\prime} C_{k}\left(\pi_{l} B^{l}\right)-\frac{1}{2 u} \varepsilon_{k l m} B^{l} \xi^{m}\right)
\end{aligned}
$$

Or, in vector notation:

$$
\begin{aligned}
& \overline{\mathbf{q}}=\frac{\sqrt{u}}{\sqrt{\chi}}\left(\bar{\xi}+\gamma^{\prime} \overline{\mathbf{B}}(\underline{\mathbf{C}} \cdot \bar{\xi})+\frac{1}{2 u} \underline{\pi} \times \underline{\mathbf{C}}\right) ; \\
& \underline{\mathbf{p}}=\frac{\sqrt{u}}{\sqrt{\chi}}\left(\underline{\pi}+\gamma^{\prime} \underline{\mathbf{C}}(\underline{\pi} \cdot \overline{\mathbf{B}})-\frac{1}{2 u} \overline{\mathbf{B}} \times \bar{\xi}\right),
\end{aligned}
$$


where

$$
\gamma^{\prime}=\frac{\sqrt{\chi}-\sqrt{u}}{\vartheta \sqrt{u}}
$$

Again, for sake of simplicity, we consider a configuration space which is Euclidean $Q=\mathbf{E}^{3}$ with metric $\langle\overline{\mathbf{v}} ; \overline{\mathbf{w}}\rangle=$ $\delta_{i j} v^{i} w^{j}=(\underline{\mathbf{v}} \cdot \overline{\mathbf{w}})$ such that $v_{i}=\delta_{i j} v^{i}$. Substitution of (IV.88) in a Hamiltonian of the form (IV.2), leads to a Hamiltonian quadratic in $(\xi, \pi)$ and to a system of linear evolution equations. In the case when $\overline{\mathbf{B}}$ and $\underline{\mathbf{C}}$ point in the same direction:

$$
\overline{\mathbf{B}}=B \overline{\mathbf{e}}_{Z} ; \underline{\mathbf{C}}=C \underline{\mathbf{e}}_{Z},
$$

a particularly simple Hamiltonian is obtained. Parallel coordinates are defined by $\xi^{3}, \pi_{3}$ and transverse coordinate vectors by $\bar{\xi}_{\perp}=\bar{\xi}-\xi^{3} \overline{\mathbf{e}}_{Z}$ and $\underline{\pi}_{\perp}=\underline{\pi}-\pi_{3} \underline{\mathbf{e}}_{Z}$. Indeed, eq. (IV.88) becomes

$$
\begin{gathered}
q^{1}=\frac{\sqrt{u}}{\sqrt{\chi}}\left(\xi^{1}+\frac{1}{2 u} \pi_{2} C\right), p_{1}=\frac{\sqrt{u}}{\sqrt{\chi}}\left(\pi_{1}+\frac{1}{2 u} \xi^{2} B\right), \\
q^{2}=\frac{\sqrt{u}}{\sqrt{\chi}}\left(\xi^{2}-\frac{1}{2 u} \pi_{1} C\right), p_{2}=\frac{\sqrt{u}}{\sqrt{\chi}}\left(\pi_{2}-\frac{1}{2 u} B \xi^{1}\right), \\
q^{3}=\xi^{3}, p_{3}=\pi_{3} .
\end{gathered}
$$

The Hamiltonian is:

$$
\mathcal{H}(\xi, \pi)=\left(\frac{1}{2 m_{\perp}}\left(\underline{\pi}_{\perp}\right)^{2}+\frac{k_{\perp}}{2}\left(\bar{\xi}_{\perp}\right)^{2}\right)+\left(\frac{1}{2 m}\left(\pi_{3}\right)^{2}+\frac{k}{2}\left(\xi^{3}\right)^{2}\right)+\mathcal{H}_{\text {int }}(\xi, \pi) .
$$

The transverse degrees of freedom are seen to have a renormalised[25] mass and elasticity constant which are given by the same expressions as in (IV.18):

$$
\frac{1}{m_{\perp}}==\frac{1}{m} \frac{u}{\chi}\left(1+\frac{c^{2}}{4 u^{2}}\right) ; \kappa_{\perp}=\kappa \frac{u}{\chi}\left(1+\frac{b^{2}}{4 u^{2}}\right),
$$

where

$$
b=\frac{B}{\sqrt{m \kappa}} ; c=C \sqrt{m \kappa} .
$$

The fields $\overline{\mathbf{B}}$ and $\mathbf{C}$ induce a sort of magnetic moment interaction along the $Z$-axis with the same Larmor frequency as before:

$$
\widetilde{\mathcal{H}}_{\text {ind }}(\xi, \pi)=-\omega_{L}^{\prime} \Lambda_{3}
$$

where $\Lambda_{3}=\xi^{1} \pi_{2}-\xi^{2} \pi_{1}$. Acrtually, the condition (IV.91) reduces the $(N=3)$ case to a sum $(N=2) \oplus(N=1)$. The three relevant frequencies of our oscilator are:

$$
\omega_{3}=\sqrt{k / m} ; \omega_{\perp}=\sqrt{k_{\perp} / m_{\perp}} ; \omega_{L}^{\prime}=\frac{1}{\chi} \omega_{0}(b-c) .
$$

The spectrum of the quantum Hamiltonian is easily obtained as

$$
E\left(n_{(+)}, n_{(-)}, n_{3}\right)=\hbar \omega_{(+)}\left(n_{(+)}+1 / 2\right)+
$$

$\hbar \omega_{(-)}\left(n_{(-)}+1 / 2\right)+\hbar \omega_{3}\left(n_{3}+1 / 2\right)$,

where $n_{( \pm)}, n_{3}$ are nonnegative integers. Corresponding eigenvectors are denoted by $\mid n_{(+)}, n_{(-)}, n_{3}>$.

\section{SYMMETRIES}

For Euclidean configuration space $Q \equiv \mathbf{E}^{N}$, with metric $\delta_{i j}$, an infinitesimal rotation is written as:

$$
\varphi: q^{i} \rightarrow q^{i}=q^{i}+\frac{1}{2} \delta \varepsilon^{\alpha \beta}\left(M_{\alpha \beta}\right)_{j}^{i} q^{j},
$$

where $\left(M_{\alpha \beta}\right)_{j}^{i}=\delta_{\alpha}^{i} \delta_{\beta j}-\delta_{\beta}^{i} \delta_{\alpha j}$ are the generators of the rotation group obeying the Lie algebra relations:

$$
\left[M_{\alpha \beta}, M_{\mu v}\right]=-\delta_{\alpha \mu} M_{\beta v}+\delta_{\alpha v} M_{\beta \mu}-\delta_{\beta v} M_{\alpha \mu}+\delta_{\beta \mu} M_{\alpha v} .
$$

This induces the push forward in $T^{*}(Q)$ :

$$
\begin{aligned}
& \widetilde{\varphi}: T^{*}(Q) \rightarrow T^{*}(Q):\left(q^{i}, p_{k}\right) \rightarrow\left(q^{\prime i}, p^{\prime}{ }_{k}\right), \\
& q^{\prime i}=q^{i}+\frac{1}{2} \delta \varepsilon^{\alpha \beta}\left(M_{\alpha \beta}\right)_{j}^{i} q^{j} ; \\
& p^{\prime}{ }_{k}=p_{k}-\frac{1}{2} \delta \varepsilon^{\alpha \beta} p_{l}\left(M_{\alpha \beta}\right)_{k}^{l} .
\end{aligned}
$$

In a basis[26] $\left\{\mathbf{e}_{\alpha \beta}\right\}$ of $\mathcal{L}(S O(N))$, let $\mathbf{u}=(1 / 2) \mathbf{e}_{\alpha \beta} u^{\alpha \beta}$ denote a generic element. With $\mathcal{R}(\mathbf{u})=\exp \left\{\frac{1}{2} u^{\alpha \beta} M_{\alpha \beta}\right\}$, finite rotations are written as

$$
q^{i} \rightarrow q^{\prime i}=\mathcal{R}(\mathbf{u})_{j}^{i} q^{j} ; p_{k} \rightarrow p^{\prime}{ }_{k}=p_{l} \mathcal{R}^{-1}(\mathbf{u})_{k}^{l} .
$$

The vector field $\mathbf{X}_{\mathbf{u}}$ (see appendix $\mathbf{A}$ ) is given by its components:

$$
\left(X_{\mathbf{u}}\right)^{i}=\frac{1}{2} u^{\alpha \beta}\left(M_{\alpha \beta}\right)_{j}^{i} q^{j} ;\left(X_{\mathbf{u}}\right)_{k}=-\frac{1}{2} u^{\alpha \beta} p_{l}\left(M_{\alpha \beta}\right)_{k}^{l} .
$$

It conserves the canonical symplectic potential and two-form:

$$
\mathcal{L}_{X_{\mathbf{u}}} \theta_{0}=0 ; \mathcal{L}_{X_{\mathbf{u}}} \omega_{0}=0 \text {. }
$$


The action is in fact Hamiltonian for the canonical symplectic structure. With the notation of appendix $\mathbf{A}$, we have

$$
\begin{aligned}
& \mathbf{X}_{\mathbf{u}}=\omega_{0}^{\sharp}(\mathbf{d} \Xi(\mathbf{u})), \\
& \Xi(\mathbf{u})=\frac{1}{2} u^{\alpha \beta} g_{\alpha \beta}^{0}(q, p), \\
& g^{0}: T^{*}(Q) \rightarrow \mathcal{L}^{*}(S O(N)):(q, p) \rightarrow \frac{1}{2} g_{\alpha \beta}^{0}(q, p) \mathbf{e}^{\alpha \beta}, \\
& g_{\alpha \beta}^{0}(q, p)=p_{k}\left(M_{\alpha \beta}\right)^{k}{ }_{j} q^{j} .
\end{aligned}
$$

In terms of the momenta $g_{\alpha \beta}^{0}$, the rotation (V.98) reads

$$
\delta q^{i}=\frac{1}{2} \delta \varepsilon^{\alpha \beta}\left\{q^{i}, g_{\alpha \beta}^{0}\right\}_{0} ; \delta p_{k}=\frac{1}{2} \delta \varepsilon^{\alpha \beta}\left\{p_{k}, g_{\alpha \beta}^{0}\right\}_{0} .
$$

The Lie algebra relations (V.99) become Poisson brackets:

$$
\left\{g_{\alpha \beta}^{0}, g_{\mu v}^{0}\right\}_{0}=-\delta_{\alpha \mu} g_{\beta v}^{0}+\delta_{\alpha v} g_{\beta \mu}^{0}-\delta_{\beta v} g_{\alpha \mu}^{0}+\delta_{\beta \mu} g_{\alpha v}^{0} .
$$

Naturally, for the modified symplectic structure (III.1), the action (V.100) will be symplectic if, and only if, the magnetic fields obey:

$$
\begin{aligned}
F_{k l}(q) & =F_{i j}(\mathcal{R}(\mathbf{u}) q)(\mathcal{R}(\mathbf{u}))^{i}{ }_{k}(\mathcal{R}(\mathbf{u}))^{j}{ }_{l}, \\
G^{k l}(p) & =\left(\mathcal{R}^{-1}(\mathbf{u})\right)^{k}{ }_{i}\left(\mathcal{R}^{-1}(\mathbf{u})\right)_{j}^{l} G^{i j}\left(p \mathcal{R}^{-1}(\mathbf{u} \gamma) \mathrm{V} .\right.
\end{aligned}
$$

For constant magnetic fields, this holds if $\mathcal{R}(\mathbf{u})$ belongs to the intersection of the isotropy groups of $\mathbf{F}$ and $\mathbf{G}$, which, in three dimensions, is not empty if both magnetic fields are along the same axis. A rotation along this "z-axis" is then symplectic. However, in general it will not be Hamiltonian and there will be no momentum $g_{Z}$ such that $\delta q=\left\{q, g_{Z}\right\}$. Again the discussion simplifies when one of the charges $r$ or $e$ vanishes. If the potentials $\mathbf{A}$ or $\widetilde{\mathbf{A}}$ are invariant under $\mathcal{R}(\mathbf{u})$, then the action is Hamiltonian[27] with momentum defined by the symplectic potentials (III.13) or (III.18) as

$$
\langle\mathcal{g}(q, p) \mid \mathbf{u}\rangle=\left\langle\boldsymbol{\theta}_{(e, 0)} \mid X_{\mathbf{u}}\right\rangle \text { or }\left\langle\boldsymbol{\theta}_{(0, r)} \mid X_{\mathbf{u}}\right\rangle .
$$

Obviously there is always an $S O(N)$ group action on the $(\xi, \pi)$ coordinates which is Hamiltonian with respect to (III.1) and momentum given by:

$$
g_{\alpha \beta}(\xi, \pi)=\pi_{k}\left(M_{\alpha \beta}\right)^{k}{ }_{j} \xi^{j} .
$$

However, the hamiltonian (IV.2), looking apparently $S O(N)$ symmetric, is explicitely seen not to be so when expressed in the $(\xi, \pi)$ variables.

\section{FINAL COMMENTS}

The symplectic structure in cotangent space, $T^{*}(Q) \stackrel{\kappa}{\rightarrow} Q$, was modified through the introduction of a closed two-form $\mathbf{F}$ on $T^{*} Q$, which has the geometic meaning of the pull-back of the magnetic field $F$, a closed two-form on $Q: \mathbf{F}=\kappa^{*}(F)$. A first caveat warns us that the other closed two-form $\mathbf{G}$ does not have such an intrinsic interpretation. Indeed, it is obvious that a mere change of coordinates in $Q$ will spoil the form (III.1) of $\omega$. This means that our approach must be restricted to configuration spaces with additional properties, which have to be conserved by coordinate changes. The most simple example is a flat linear[28] space $Q=\mathbf{E}^{N}$, when (III.1) is assumed to hold in linear coordinates. Obviously, a linear change in coordinates will then conserve this particular form. Although the restriction to constant fields $\mathbf{F}$ and $\mathbf{G}$ is a severe limitation[29], it allowed us to find explicit Darboux coordinates (IV.8) when $N=2$ and (IV.81) when $N=3$.

Finally, when $\operatorname{det}\{\mathbf{1}-r \mathbf{G} e \mathbf{F}\}=0$, the closed two-form $\omega$ is degenerate with constant rank and defines a pre-symplectic structure on $T^{*}(Q)$. Its null-foliation decomposes $T^{*}(Q)$ in disjoint leaves and on the space of leaves, $\omega$ projects to a unique symplectic two-form. In two dimensions, the representations of the corresponding quantum algebra in Hilbert space and its reduction in the degeneracy case were studied in $[11-14,18]$

\section{APPENDIX A: ESSENTIAL SYMPLECTIC MECHANICS}

Let $\{\mathcal{M}, \omega\}$ be a symplectic manifold with symplectic structure defined by a two-form $\omega$ which is closed, $\mathbf{d} \omega=$ 0 , and nondegenerate such that the induced mapping $\omega^{b}$ : $T(\mathcal{M}) \rightarrow T^{*}(\mathcal{M}): \mathbf{X} \rightarrow \iota_{\mathbf{X}} \omega$ has an inverse $\omega^{\sharp}: T^{*}(\mathcal{M}) \rightarrow$ $T(\mathcal{M}): \alpha \rightarrow \omega^{\sharp}(\alpha)$. The paradigm of a (non-compact) symplectic manifold is a cotangent bundle $T^{*}(Q)$ of a differential configuration space $Q$. In a coordinate system $\left\{q^{i}\right\}$ of $Q$, a cotangent vector may be written as $\alpha_{q}=p_{i} \mathbf{d} q^{i}$. This defines coordinates $z \Rightarrow\left\{q^{i}, p_{k}\right\}$ of points $z \in \mathcal{M} \equiv T^{*}(Q)$ and an associated holonomic basis $\left\{\mathbf{d} p_{k}, \mathbf{d} q^{i}\right\}$ of $T_{z}^{*}(\mathcal{M})$. The canonical one-form is defined as $\theta_{0} \doteq p_{i} \mathbf{d} q^{i}$. Obviously, the exact two-form $\omega_{0} \doteq-\mathbf{d} \theta_{0}=\mathbf{d} q^{i} \wedge \mathbf{d} p_{i}$ is symplectic.

To each observable, which is a differentiable function $f$ on $\{\mathcal{M}, \omega\}$, the symplectic structure associates a Hamiltonian vector field:

$$
\mathbf{X}_{f} \doteq \omega^{\sharp}(\mathbf{d} f) \quad \text { or } \quad l_{\mathbf{X}_{f}} \omega=\mathbf{d} f .
$$

Such a vector field generates a one-parameter (local) transformation group: $\mathcal{T}_{f}(t): \mathcal{M} \rightarrow \mathcal{M}: z_{0} \rightarrow z(t)$, solution of $\mathbf{d} z(t) / \mathbf{d} t=\mathbf{X}_{f}(z(t)), z(0)=z_{0}$.

In particular, the Hamiltonian $\mathcal{H}$ generates the dynamics of the associated mechanical system. With the usual interpretation of time, $\mathbf{X}_{\mathcal{H}}$ is assumed to be complete such that its flux is defined for all $t \in[-\infty,+\infty]$. Transformations, induced by an Hamiltonian vector field $\mathbf{X}_{f}$, conserve the symplectic structure[30]:

$$
\mathcal{T}_{f}(t)^{*} \omega=\omega \text { or, locally: } \mathcal{L}_{\mathbf{X}_{f}} \omega=0 .
$$

More generally, the transformations conserving the symplectic structure form the group $\operatorname{Sympl}(\mathcal{M})$ of symplectomorphisms or canonical transformations. Vector fields obeying $\mathcal{L}_{X} \omega=0$, generate canonical transformations and are called locally Hamiltonian, since [31] $\mathbf{d}_{\mathbf{X}} \boldsymbol{\omega}=0$ implies that, locally in some $U \subset \mathcal{M}$, there exists a function $f$ such that $\mathbf{d} f_{\mid U}=\left(l_{\mathbf{X}} \omega\right)_{\mid U}$. 
The Darboux theorem guarantees the existence of local charts $U \subset \mathcal{M}$ with coordinates $\left\{q^{i}, p_{k}\right\}$ such that, in each $U, \omega$ is written as:

$$
\omega_{\mid U}=\mathbf{d} q^{i} \wedge \mathbf{d} p_{i}
$$

In the natural basis $\left\{\partial / \partial \mathbf{q}^{\mathbf{i}}, \partial / \partial \mathbf{p}_{\mathbf{k}}\right\}$ of $T_{z}(\mathcal{M})$, the Hamiltonian vector fields corresponding to $f$ reads

$$
\mathbf{X}_{f}=\frac{\partial f}{\partial p_{i}} \frac{\partial}{\partial \mathbf{q}^{i}}-\frac{\partial f}{\partial q^{i}} \frac{\partial}{\partial \mathbf{p}_{i}}
$$

The Poisson bracket of two observables is defined by: $\{f, g\} \doteq \omega\left(\mathbf{X}_{f}, \mathbf{X}_{g}\right)$, with the following properties:

$$
\begin{aligned}
& \left\{f_{1}, f_{2}\right\}=-\left\{f_{2}, f_{1}\right\} \\
& \left\{f_{1}, g_{1} \cdot g_{2}\right\}=\left\{f, g_{1}\right\} \cdot g_{2}+g_{1} \cdot\left\{f, g_{2}\right\} \\
& \left\{f,\left\{g_{1}, g_{2}\right\}\right\}=\left\{\left\{f, g_{1}\right\}, g_{2}\right\}+\left\{g_{1},\left\{f, g_{2}\right\}\right\}
\end{aligned}
$$

These properties, relating the pointwise product $g_{1} \cdot g_{2}$ with the bracket $\{f, g\}$, are said to endow the set of differentiable functions on $\mathcal{M}$ with the structure of a Poisson algebra $\mathcal{P}(\mathcal{M})$. In a coordinate system $\left(z^{A}\right)$, where $\omega=\frac{1}{2} \omega_{A B} \mathbf{d} z^{A} \wedge$ $\mathbf{d} z^{B}$, it is given by:

$$
\{f, g\}=\frac{\partial f}{\partial z^{A}} \Lambda^{A B} \frac{\partial g}{\partial z^{B}},
$$

where $\Lambda$ is minus $\omega^{-1}$. In Darboux coordinates it reads:

$$
\{f, g\}_{0}=\frac{\partial f}{\partial q^{i}} \frac{\partial g}{\partial p_{i}}-\frac{\partial f}{\partial p_{i}} \frac{\partial g}{\partial q^{i}} .
$$

The Poisson brackets of the Darboux coordinates themselves are:

$\left\{q^{i}, q^{j}\right\}_{0}=0,\left\{q^{i}, p_{l}\right\}_{0}=\delta_{l}^{i},\left\{p_{k}, q^{j}\right\}_{0}=-\delta_{k}^{j},\left\{p_{k}, p_{l}\right\}_{0}=0$

The dynamical evolution of an observable is given by:

$$
\frac{\mathbf{d} f}{\mathbf{d} t}=\overrightarrow{\mathbf{X}}_{\mathcal{H}}(f)=l_{\mathbf{X}_{\mathcal{H}}} \mathbf{d} f=l_{\mathbf{X}_{\mathcal{H}}} \mathbf{X}_{f} \omega=\omega\left(\mathbf{X}_{f}, \mathbf{X}_{\mathcal{H}}\right)=\{f, \mathcal{H}\} .
$$

A Lie group $G$ acts as a symmety group on a symplectic manifold $\mathcal{M}$, if there is a group homomorphism $\mathcal{T}: G \rightarrow$ $\operatorname{Sympl}(\mathcal{M}): g \rightarrow \mathcal{T}(g)$. An infinitesimal action defined by a Lie algebra element $\mathbf{u} \in \mathcal{G}$ is given by the locally Hamiltonian vector field

$$
\mathbf{X}_{\mathbf{u}}(z)=\frac{d}{d t}(\mathcal{T}(\exp (t \mathbf{u})) z)_{\mid t=0} .
$$

When each $\mathbf{X}_{\mathbf{u}}$ is Hamiltonian, the group action is said to be almost Hamiltonian and $\{\mathcal{M}, \omega\}$ is called a symplectic $G$ space. In such a case, a linear map $\Xi: \mathcal{G} \rightarrow \mathcal{P}(\mathcal{M}): \mathbf{u} \rightarrow$ $\Xi(\mathbf{u})$ can always be constructed such that $\mathbf{X}_{\mathbf{u}}=\omega^{\sharp}(\mathbf{d} \Xi(\mathbf{u}))$. When there is a $\Xi$ which is also a Lie algebra homomorphism: $\Xi([\mathbf{u}, \mathbf{v}])=\{\Xi(\mathbf{u}), \Xi(\mathbf{v})\}$, the group is said to have a Hamiltonian action and $\{\mathcal{M}, \omega, \Xi\}$ is called a Hamiltonian
$G$-space. Since $\Xi$ is linear in $\mathcal{G}$, it defines a momentum mapping $\mathcal{I}$ from $\mathcal{M}$ to the dual $\mathcal{G}^{*}$ of the Lie algebra defined by: $\langle\mathcal{I}(z) \mid \mathbf{u}\rangle=\Xi(\mathbf{u}, z)$. When $\mathscr{M}$ is a Hamiltonian $G$-space, the momentum mapping is equivariant under the action of $G$ on $\mathcal{M}$ and its co-adjoint action on $\mathcal{G}^{*}$.

In general there may be topological obstructions to such a Lie algebra homomorphism. However, when $G$ acts on $Q$ : $\varphi: G \rightarrow \operatorname{Diff}(Q): g \rightarrow \varphi(g): q \rightarrow q^{\prime}=\varphi(g) q$, the action is extended to a symplectic action in $\left\{\mathcal{M}=T^{*}(Q), \omega_{0}\right\}$ : $\widetilde{\varphi}: G \rightarrow \operatorname{Sympl}(\mathcal{M}): g \rightarrow \widetilde{\varphi}(g):(q, p) \rightarrow\left(q^{\prime}, p^{\prime}\right)$, where $p^{\prime}$ is defined by $p=(\varphi(g))_{\mid q}^{*} p^{\prime}$. It follows that $\widetilde{\varphi}(g)^{*} \theta_{0}=$ $\theta_{0} ; \widetilde{\varphi}(g)^{*} \omega_{0}=\omega_{0}$. The infinitesimal action is given by $\mathbf{X}_{\mathbf{u}}(z)=(\mathbf{d} \widetilde{\varphi}(\exp (t \mathbf{u})) z / d t)_{\mid t=0}$ and $\mathcal{L}_{\mathbf{X}_{\mathbf{u}}} \theta_{0}=0 ; \mathcal{L}_{\mathbf{X}_{\mathbf{u}}} \omega_{0}=0$. From $\omega_{0}^{b}\left(\mathbf{X}_{\mathbf{u}}\right)=\mathbf{d}\left\langle\theta_{0} \mid \mathbf{X}_{\mathbf{u}}\right\rangle$, it follows that the action is almost Hamiltonian with $\Xi(\mathbf{u})=\left\langle\theta_{0} \mid \mathbf{X}_{\mathbf{u}}\right\rangle$. Moreover, since $\left\langle\theta_{0} \mid \mathbf{X}_{[\mathbf{u}, \mathbf{v}]}\right\rangle=\omega_{0}\left(\mathbf{X}_{\mathbf{u}}, \mathbf{X}_{\mathbf{v}}\right)=\{\Xi(\mathbf{u}), \Xi(\mathbf{v})\}$, the action is Hamiltonian and $\left\{T^{*}(Q), \omega_{0}, \Xi\right\}$ is a Hamiltonian $G$-space.

\section{APPENDIX B: PRESYMPLECTIC MECHANICS}

A manifold $\mathcal{M}_{1}$, endowed with a closed but degenerate[32] 2 -form $\omega$, with constant rank, is said to be presymplectic. The mapping $\omega^{b}$ has a nonvanishing kernel, given by those nonzero vector fields $\mathbf{X}_{0}$ obeying $\omega^{b}\left(\mathbf{X}_{0}\right) \doteq \iota_{\mathbf{X}_{0}} \omega=0$. The fundamental dynamical equation

$$
\omega^{b}(\mathbf{X})=\mathbf{d} \mathcal{H},
$$

has then a solution if

$$
\left\langle\mathbf{d} \mathcal{H} \mid \mathbf{X}_{0}\right\rangle=0 \quad ; \forall \mathbf{X}_{0} \in \mathcal{K} \operatorname{er}\left(\omega^{b}\right)
$$

If this is nowhere satisfied on $\mathcal{M}_{1}$, the hamiltonian $\mathcal{H}$ does not define any dynamics on $\mathcal{M}_{1}$. When (B.2) is identically satisfied, a particular solution $\mathbf{X}_{P}$ of (B.1) is defined in the entire manifold $\mathcal{M}_{1}$ and so is the general solution obtained summing the general solution of the homogeneous equation $l_{\mathbf{X}_{0}} \omega=0$, i.e $\mathbf{X}_{G}=\mathbf{X}_{P}+\mathbf{X}_{0}$, which will contain arbitrary functions. When (B.2) is satisfied for some points $z \in \mathcal{M}_{1}$, we shall asssume they form a submanifold, called the secondary constrained submanifold with injection $l_{2}: \mathcal{M}_{2} \hookrightarrow \mathcal{M}_{1}$. The particular solution $\mathbf{X}_{P}$ of (B.1) is now defined in $\mathcal{M}_{2}$ and so is the general solution $\mathbf{X}_{G}$. Requiring that $\mathbf{X}_{G}$ conserves the constraints amounts to ask that $\mathbf{X}_{G}$ is tangent to $\mathcal{M}_{2}$ :

$$
\mathbf{X}_{G}=\iota_{2 \star}\left(\mathbf{X}_{2}\right) ; \mathbf{X}_{2} \in \Gamma\left(\mathcal{M}_{2}, T \mathcal{M}_{2}\right)
$$

Again, when there are no points where this tangency condition is satisfied, (B.1) is meaningless. Another possibility is that some of the arbitrary functions in $\mathbf{X}_{0}$ become determined and the tangency condition is obeyed on the entire $\mathcal{M}_{2}$. The general solution then still contains some arbitrary functions. Finally it may happen that the conditions (B.3) are only satisfied on some $\mathcal{M}_{3}$ with $\iota_{3}: \mathcal{M}_{3} \hookrightarrow \mathcal{M}_{2}$. The story then goes on until one of the first two alternatives are reached. 
[1] J. von Neumann, Math. Annalen 104, 570 (1931).

[2] R. Peierls, Z. Phys. 80,763 (1933).

[3] H. S. Snyder, Phys. Rev. 71, 38 (1947).

[4] A. Messiah, Mécanique Quantique I, Dunod,1962.

[5] J-M. Souriau, Structure des systèmes dynamiques, Dunod, 1970.

[6] R. Abraham and J. E. Marsden, Foundations of Mechanics, Benjamin, 1978

[7] M. J. Gotay, J. M. Nester, and G. Hinds, J. Math. Phys. 19, 2388 (1978).

[8] A.P. Balachandran, G. Marmo, B-S. Skagerstam, and A. Stern, Gauge Symmetries and Fibre Bundles, Lect. Notes in Physics 188 (1983).

[9] V. Guillemin and S. Sternberg, Symplectic Tecniques in Physics, Cambridge University Press, 1984.

[10] S. Doplicher, K. Fredenhagen, and J. Roberts, Comm. Math. Phys. 172, 187 (1995).

[11] C. Duval and P. A. Horváthy, Phys. Lett. B 479,284 (2000).

[12] V. P. Nair and A. Polychronakos, Phys. Lett. B 505, 267 (2001).

[13] B. Morariu, and A. Polychronakos, Nucl. Phys. B 610, 531( 2001) and Nucl. Phys. B 634, 326 (2002).

[14] P. A. Horváthy, Ann. Phys. 299, 128 (2002).

[15] A. E. F. Djemaï and H. Smail, arXiv:hep-th/0309006(2003).

[16] A. Bérard and H. Mohrbach, arXiv:hep-th/0310167(2003) and arXiv:hep-th/0404165(2004)

[17] A. P. Balachandran, T. R. Govindarajan, C. Molina, and P. Teotonio-Sobrinho, arXiv:hep-th/0406125, (2004).

[18] P. A. Horváthy and M. S. Plyushchay, Phys. Lett. B 595, 547 (2004).

[19] See e.g. [8]
[20] Well known in symplectic mechanics, see e.g.[5, 6, 9].

[21] Observe that $\Phi_{k}^{\ell}=\delta_{k}{ }^{\ell}-e \mathbf{F}_{k j} r \mathbf{G}^{j \ell}$ and $\Psi^{i}{ }_{j}=\delta^{i}{ }_{j}-r \mathbf{G}^{i \ell} e \mathbf{F}_{\ell j}$ are mutually transposed and that the matrices $\Psi^{k}{ }_{j} r \mathbf{G}^{j \ell}=$ $r \mathbf{G}^{k j} \Phi_{j}^{\ell}$ and $\Phi_{k}^{j} e \mathbf{F}_{j \ell}=e \mathbf{F}_{k j} \Psi^{j}{ }_{\ell}$ are antisymmetric.

[22] In the limit $\chi \rightarrow 0$, we have $m^{\prime} \omega_{0}^{\prime}=\sqrt{m^{\prime} \kappa^{\prime}} \rightarrow|B|$.

[23] Recall that with complex variables $q=q^{1}+\mathbf{i} q^{2}$, the diferentials $d q=d q^{1}+\mathbf{i} d q^{2}$ and $d q^{\dagger}=d q^{1}-\mathbf{i} d q^{2}$ have local dual vector fields $\left\{\partial / \partial q=\left(\partial / \partial q^{1}-\mathbf{i} \partial / \partial q^{2}\right) / 2 ; \partial / \partial q^{\dagger}=\left(\partial / \partial q^{1}+\right.\right.$ $\left.\mathbf{i} \partial / \partial q^{2}\right) / 2$ and similarly for the $p=p_{1}+\mathbf{i} p_{2}$ variables.

[24] The $(N=3)$ case wil only be examined in the nondegenerate case $\chi>0$

[25] Due to $\kappa^{2}+\kappa^{\prime 2}(r C)^{2}(e B)^{2}+2 \kappa \kappa^{\prime} r C e B=1$, the mass and elastic constant of the $z$ degrees of freedom, as expected, are not renormalised.

[26] with dual basis $\left\{\mathbf{e}^{\alpha \beta}\right\}$ in $\mathcal{L}^{*}(S O(N))$.

[27] Exercise 4.2A in [6], defining a (generalized) Poincaré momentum.

[28] Quantum mechanics on a noncommutative shere $S^{2}$ and on general noncommutative Riemann surfaces was examined in $([12,13]$.

[29] In the case $e=0$, Darboux coordinates are given by (III.17) and in [16] such model was considered with the possibility of having a monopole in $p$-space!

[30] $\mathcal{T}_{f}(t)^{*}$ denotes the pull-back of $\mathcal{T}_{f}(t)$ and $\mathcal{L}$ is the Lie derivative along $\mathbf{X}_{f}$.

[31] We use $\mathcal{L}_{\mathbf{X}}=\mathbf{d} l_{\mathbf{X}}+{ }_{\mathbf{X}} \mathbf{d}$ on differential forms.

[32] $\mathcal{M}_{1}$ is the primary constrained manifold, arising e.g. from a degenerate Lagrangian. 